\title{
Formal Pseudodifferential Operators in One and Several Variables, Central Extensions, and Integrable Systems
}

\author{
Jarnishs Beltran ${ }^{1}$ and Enrique G. Reyes ${ }^{2}$ \\ ${ }^{1}$ Centro de Investigación en Complejidad Social (CICS), Facultad de Gobierno, Universidad del Desarrollo, 7610658 Santiago, Chile \\ ${ }^{2}$ Departamento de Matemática y Ciencia de la Computación, Universidad de Santiago de Chile, Casilla 307, Correo 2, Santiago, Chile \\ Correspondence should be addressed to Enrique G. Reyes; e_g_reyes@yahoo.ca
}

Received 22 February 2015; Accepted 19 May 2015

Academic Editor: Hagen Neidhardt

Copyright (c) 2015 J. Beltran and E. G. Reyes. This is an open access article distributed under the Creative Commons Attribution License, which permits unrestricted use, distribution, and reproduction in any medium, provided the original work is properly cited.

We review some aspects of the theory of Lie algebras of (twisted and untwisted) formal pseudodifferential operators in one and several variables in a general algebraic context. We focus mainly on the construction and classification of nontrivial central extensions. As applications, we construct hierarchies of centrally extended Lie algebras of formal differential operators in one and several variables, Manin triples and hierarchies of nonlinear equations in Lax and zero curvature form.

\section{Introduction}

This paper is on some cohomological aspects of Lie algebras of formal pseudodifferential operators in one and several independent variables, motivated by previous works on algebras of importance for integrable systems and symplectic geometry such as the Lie algebra of vector fields on the circle and their deformations $[1,2]$ or the Lie algebra of differential operators; see, for example, [3-6].

A very well-known infinite-dimensional Lie algebra of interest for physics is the Virasoro algebra [7]. This algebra is a one-dimensional central extension of the Lie algebra of vector fields on the circle (also called centerless Virasoro algebra) which also appears naturally in applications. We mention, as a recent example, that the centerless Virasoro algebra can be realized as an algebra of nonlocal symmetries for the CamassaHolm and Hunter-Saxton equations $[8,9]$.

Now, the Lie algebra of vector fields on the circle is included naturally in the Lie algebra of differential operators on the circle. This algebra, in turn, is included in the Lie algebra of formal pseudodifferential operators on the circle which has been studied very carefully, for example, by Dickey [5] and Adler [10], in connection with the algebraic and geometric theory of the famous Korteweg-de Vries (KdV) equation and other integrable systems. Moreover, there exist nontrivial twisted versions of these works related to "twisted" and "quantum" analogs of classical integrable systems; see, for instance, [11-14]. It is certainly reasonable to consider formal pseudodifferential operators as a general arena for integrable systems.

Our aim in this work is to study central extensions of Lie algebras of formal pseudodifferential operators in a general algebraic setting and to apply this study to the construction of hierarchies of centrally extended Lie algebras, Manin triples [15], and nonlinear integrable equations in one and several independent variables.

We mention four examples of relevant central extensions. Centerless Virasoro has a unique central extension; see [3]. Also, a 2-cocycle of the algebra of differential operators on the circle was constructed in [6], and a 2-cocycle of the algebra of pseudodifferential operators on the circle was constructed by Kravchenko and Khesin using logarithms; see $[16,17]$. Finally, a 2-cocycle for a quantum analog of the algebra of pseudodifferential operators was considered in [11].

In this paper we consider algebraic versions of these results, and we present classifications of central extensions. In particular, we show that many of the constructions of cocycles appearing in the literature (see, for instance, [17] or [4]) are valid well beyond their original framework.

We have divided our work in three main sections. 
In Section 2 we introduce the main objects considered in this work. We define formal pseudodifferential operators (or, "pseudodifferential symbols") in one variable on an arbitrary associative and commutative algebra, and we construct the Kravchenko-Khesin logarithmic 2-cocycle in this general context. We also construct a hierarchy of centrally extended Lie algebras of differential operators via the logarithmic 2cocycle. This construction generalizes a theorem by Khesin [18] on hierarchies of Lie algebras of differential operators on the circle. Finally, motivated by $[11,19,20]$ and the later paper [12], we consider twisted pseudodifferential symbols on arbitrary associative and commutative algebras and, in analogy with the untwisted case, we construct central extensions and hierarchies of twisted centrally extended algebras.

In Section 3 we consider pseudodifferential symbols in several independent variables on an arbitrary associative and commutative algebra. Our main motivation for considering the several variables case in detail is the relative absence of examples of integrable equations in this context. Indeed, besides the equations of the standard KP hierarchy [5] and their cousins, there are not very many general constructions of integrable equations in several independent variables. Important exceptions are the equations introduced by Tenenblat and her coworkers (see [21] and references therein) and the hierarchies considered by Parshin, [22]. We construct central extensions in the several variables case using logarithmic cocycles, and we also exhibit hierarchies of Lie algebras of differential operators in several independent variables admitting central extensions. We then consider the work [23] by Dzhumadil'daev, in which he presents a classification of central extensions. The paper [23] is quite technical and it requires a very careful and critical reading, and so we have decided to explain how to prove the main result of [23] using an inductive argument. We present a full inductive proof of Dzhumadil'daev's theorem using some technical homological tools elsewhere; see [24].

Finally, in Section 4 we introduce Manin triples, we define double extensions for the algebras of (twisted) pseudodifferential symbols in one and several independent variables and, using a general algebraic theorem [25], we construct Manin triples for these algebras, thereby putting [17, 26-29] in a very general framework. We also apply our algebraic results to the construction of integrable systems in one and several independent variables, roughly following the techniques of $[4,11,22]$.

\section{The Algebra of Formal Pseudodifferential Symbols in One Variable}

2.1. Basic Definitions and Preliminary Results. Let $\mathscr{A}$ be an associative and commutative algebra, and let $\delta: \mathscr{A} \rightarrow \mathscr{A}$ be a derivation on $\mathscr{A}$; that is, $\delta$ is a linear map such that $\delta(a b)=a \delta(b)+\delta(a) b$ for all $a, b \in \mathscr{A}$. The algebra of formal differential symbols DO is generated by $\mathscr{A}$ and a symbol $\xi$ with the relation for all $a \in \mathscr{A}$. The algebra $\mathscr{A}$ is a subalgebra of DO and we can prove inductively that

$$
\xi^{n} \circ a=\sum_{j=0}^{n}\left(\begin{array}{l}
n \\
j
\end{array}\right) \delta^{j}(a) \xi^{n-j}
$$

for all $a \in \mathscr{A}$ and $n \geq 0$.

We extend the algebra DO to obtain the algebra of pseudodifferential symbols $\Psi \mathrm{DO}$ by introducing differentiations with negative exponents. A general element of $\Psi D O$ is a formal series $P$ of the form

$$
P=\sum_{-\infty}^{n} a_{i} \xi_{i}, \quad \text { where } a_{i} \in \mathscr{A} .
$$

We set

$$
\xi^{-1} \circ a=\sum_{i=0}^{\infty}(-1)^{i} \delta^{i}(a) \xi^{-1-i}
$$

and so (2) generalizes to

$$
\xi^{n} \circ a=\sum_{j=0}^{\infty}\left(\begin{array}{l}
n \\
j
\end{array}\right) \delta^{j}(a) \xi^{n-j}
$$

for all $n \in \mathbb{Z}$, in which the binomial coefficient is defined by

$$
\left(\begin{array}{l}
n \\
j
\end{array}\right)=\frac{n(n-1) \cdots(n-j+1)}{j !}
$$

for $n, j \in \mathbb{Z}$. If $P_{1}=\sum_{i=-\infty}^{M} a_{i} \xi^{i}$ and $P_{2}=\sum_{j=-\infty}^{N} b_{j} \xi^{j}$, then

$$
P_{1} \circ P_{2}=\sum_{k=0}^{\infty} \frac{1}{k !} \frac{\partial^{k}}{\partial \xi^{k}}\left(P_{1}\right) \delta^{k}\left(P_{2}\right) .
$$

The Lie algebra structure on $\Psi D O$ is given by the usual commutator $[A, B]=A \circ B-B \circ A$, so that, for instance,

$$
\left[\xi^{\alpha}, a \xi^{n}\right]=\sum_{j=1}^{\infty}\left(\begin{array}{l}
n \\
j
\end{array}\right) \delta^{j}(a) \xi^{\alpha+n-j} .
$$

Lemma 1. For any nonnegative integer $m$ and $a, b \in \mathscr{A}$, we have

$$
\delta^{m}(a b)=\sum_{j=0}^{m}\left(\begin{array}{c}
m \\
j
\end{array}\right) \delta^{m-j}(a) \delta^{j}(b) .
$$

Proof. Proof is by induction.

Let $\tau: \mathscr{A} \rightarrow \mathbb{C}$ be a $\delta$-invariant trace on $\mathscr{A}$; that is, $\tau$ is a linear map satisfying $\tau(a b)=\tau(b a)$ and $\tau(\delta(a))=0$ for all $a, b \in \mathscr{A}$. For example, if $\mathscr{A}=\operatorname{Diff}\left(S^{1}\right), \delta=d / d x$, the linear functional $\tau: \operatorname{Diff}\left(S^{1}\right) \rightarrow \mathbb{C}$ given by $\tau(f)=\int_{S^{1}} f(x) d x$ is a $\delta$-invariant trace.

Lemma 2. Let $\mathscr{A}$ be an algebra, $\delta$ a derivation on $\mathscr{A}, \tau$ a $\delta$ invariant trace on $\mathscr{A}$, and $m$ a positive integer. Then we can "integrate by parts"; that is, for all $a, b \in \mathscr{A}$, we have

$$
\tau\left(b \delta^{m}(a)\right)=(-1)^{m} \tau\left(a \delta^{m}(b)\right) .
$$


Proof. We have that $\delta(a b)=a \delta(b)+\delta(a) b$, and, so, $0=$ $\tau(\delta(a b))=\tau(a \delta(b))+\tau(\delta(a) b)$; then $-\tau(a \delta(b))=\tau(\delta(a) b)$. We now proceed by induction on $m$.

Proposition 3. Let $\mathscr{A}$ be an algebra, $\delta$ a derivation on $\mathscr{A}$, and $\tau$ a $\delta$-invariant trace. Then the linear map res : $\Psi D O \rightarrow \mathbb{C}$ defined by

$$
\operatorname{res}\left(\sum_{i=-\infty}^{M} a_{i} \xi^{i}\right)=\tau\left(a_{-1}\right)
$$

is a trace on $\Psi D O$; that is, res is linear and it satisfies $\operatorname{res}(A B)=$ $\operatorname{res}(B A)$ for all $A, B \in \Psi D O$. This is the Adler-Manin noncommutative residue introduced in $[10,30]$.

Proof. We use the elementary identity

$$
\left(\begin{array}{l}
a \\
b
\end{array}\right)=(-1)^{b}\left(\begin{array}{c}
b-a-1 \\
b
\end{array}\right)
$$

for $a<0$ and $b \geq 0$. Since res is linear, it is sufficient to show that for any $a, b \in \mathscr{A}$ and $m, n \in \mathbb{Z}$

$$
\operatorname{res}\left(a \xi^{n} \circ b \xi^{m}\right)=\operatorname{res}\left(b \xi^{m} \circ a \xi^{n}\right) \text {. }
$$

We consider several cases.

Let $m \cdot n \geqslant 0$

$$
\begin{aligned}
\operatorname{res}\left(a \xi^{n} \circ b \xi^{m}\right) & =\sum_{j=0}^{\infty} \operatorname{res}\left(\left(\begin{array}{l}
n \\
j
\end{array}\right) a \delta^{j}(b) \xi^{m+n-j}\right) \\
& =\tau\left(\left(\begin{array}{c}
n \\
m+n+1
\end{array}\right) a \delta^{m+n+1}(b)\right)
\end{aligned}
$$

and this is identically zero since we obtain the coefficient of $\xi^{-1}$ when $j=m+n+1$, and $\left(\begin{array}{c}n \\ m+n+1\end{array}\right)=0$. Similarly,

$$
\operatorname{res}\left(b \xi^{m} \circ a \xi^{n}\right)=\tau\left(\left(\begin{array}{c}
m \\
m+n+1
\end{array}\right) a \delta^{m+n+1}(b)\right)=0 .
$$

Now we let $m, n<0$; then $m+n-j \leq n+m<-1$ ( $j$ a positive integer), and

$$
\operatorname{res}\left(a \xi^{n} \circ b \xi^{m}\right)=\operatorname{res}\left(\sum_{j=0}^{\infty}\left(\begin{array}{l}
n \\
j
\end{array}\right) a \delta^{j}(b) \xi^{m+n-j}\right) .
$$

But, as $m+n-j<-1$, the coefficient of $\xi^{-1}$ is 0 , and then $\operatorname{res}\left(a \xi^{n} b \xi^{m}\right)=0$. Analogously we find $\operatorname{res}\left(b \xi^{m} \circ a \xi^{n}\right)=0$.

Now assume that $n \geqslant 0, m<0$. If $n+m<-1$ then

$$
\operatorname{res}\left(a \xi^{n} \circ b \xi^{m}\right)=\operatorname{res}\left(\sum_{j=0}^{\infty}\left(\begin{array}{l}
n \\
j
\end{array}\right) a \delta^{j}(b) \xi^{m+n-j}\right)=0
$$

because $n+m-j<-1-j \leq-1$ and then $n+m-j<-1$. Analogously, $\operatorname{res}\left(b \xi^{m} \circ a \xi^{n}\right)=0$.

Finally, we let $n+m \geqslant-1$; set $k=m+n$. Then,

$$
\begin{aligned}
\operatorname{res}\left(a \xi^{n} \circ b \xi^{m}\right) & =\tau\left(\left(\begin{array}{c}
n \\
k+1
\end{array}\right) a \delta^{k+1}(b)\right) \\
& =\left(\begin{array}{c}
n \\
k+1
\end{array}\right) \tau\left(a \delta^{k+1}(b)\right)
\end{aligned}
$$

and, on the other hand, (10) and (12) imply

$$
\begin{aligned}
\operatorname{res} & \left(b \xi^{m} \circ a \xi^{n}\right)=\tau\left(\left(\begin{array}{c}
m \\
k+1
\end{array}\right) b \delta^{k+1}(a)\right) \\
& =\left(\begin{array}{c}
k-n \\
k+1
\end{array}\right) \tau\left(b \delta^{k+1}(a)\right) \\
& =(-1)^{k+1}\left(\begin{array}{c}
n \\
k+1
\end{array}\right) \tau\left(b \delta^{k+1}(a)\right) \\
& =(-1)^{k+1}(-1)^{k+1}\left(\begin{array}{c}
n \\
k+1
\end{array}\right) \tau\left(a \delta^{k+1}(b)\right) \\
& =\left(\begin{array}{c}
n \\
k+1
\end{array}\right) \tau\left(a \delta^{k+1}(b)\right) .
\end{aligned}
$$

Remark 4. Our definition of residue follows the conventions of [23]. For example, if $\mathscr{A}=\operatorname{Diff}\left(S^{1}\right), \delta=d / d x$, and $\tau$ : $\operatorname{Diff}\left(S^{1}\right) \rightarrow \mathbb{C}$ is given by $\tau(f)=\int_{S^{1}} f(x) d x$, then, with our convention,

$$
\operatorname{res}(A)=\operatorname{res}\left(\sum_{i=-\infty}^{n} a_{i}(x) \partial^{i}\right)=\tau\left(a_{-1}(x)\right),
$$

which is slightly different from the notation used in [4, 16-18].

Corollary 5. The bilinear form $\langle A, B\rangle=\operatorname{res}(A \circ B)$ is adinvariant; that is, it satisfies $\langle[A, B], C\rangle=\langle A,[B, C]\rangle$.

Now, if $P=a_{n} \xi^{n}+a_{n-1} \xi^{n-1}+\cdots$ is a pseudodifferential symbol such that $a_{n} \neq 0$ and $a_{m}=0$ for all $m>n$, we say that $n$ is the order or degree of $P$. The following observation has resulted to be fundamental for the theory; see, for instance, [5, $10,17,31]$. The algebra $\Psi D O$ can be decomposed as a (vector space) direct sum $\Psi \mathrm{DO}=\mathrm{DO} \oplus \mathrm{INT}$, where

$$
\begin{aligned}
& \mathrm{DO}=\left\{\sum_{i=0}^{n} a_{i} \xi^{i} \mid a_{i} \in \mathscr{A}\right\}, \\
& \mathrm{INT}=\left\{\sum_{i=-\infty}^{-1} a_{i} \xi^{i} \mid a_{i} \in \mathscr{A}\right\} .
\end{aligned}
$$

Proposition 6. The subalgebras DO (of differential operators) and INT (of pseudodifferential symbols of order $\leq-1$ ) are isotropic subspaces of $\Psi D O$ with respect to the bilinear form defined in Corollary 5; that is, the restrictions of this form to both DO and INT vanish.

2.2. On Cohomology of Lie Algebras. Having reviewed the elementary properties of $\Psi \mathrm{DO}$, we now summarize some basic facts on the cohomology of Lie algebras in order to fix our notation. We will study the cohomology of $\Psi D O$ in Section 2.3. 
2.2.1. Basic Definitions. Suppose that $\mathfrak{g}$ is a Lie algebra and that $A$ is a module over $\mathfrak{g}$. A $q$-dimensional cochain of the algebra $\mathfrak{g}$ with coefficients in $A$ is a skew-symmetric $q$-linear functional on $g$ with values in $A$; the space of all such cochains is denoted by $C^{q}(\mathfrak{g}, A)$ and we also set $C^{0}(\mathfrak{g}, A)=A$. The differential $d=d_{q}: C^{q}(\mathfrak{g}, A) \rightarrow C^{q+1}(\mathfrak{g}, A)$ is defined by the formula

$$
\begin{aligned}
& d c\left(g_{1}, \ldots, g_{q+1}\right)=\sum_{1 \leq s<t \leq q+1}(-1)^{s+t-1} \\
& \cdot c\left(\left[g_{s}, g_{t}\right], g_{1}, \ldots, \widehat{g}_{s}, \ldots, \widehat{g}_{t}, \ldots, g_{q+1}\right) \\
& \quad+\sum_{1 \leq s \leq q+1}(-1)^{s} g_{s} c\left(g_{1}, \ldots, \widehat{g}_{s}, \ldots, g_{q+1}\right),
\end{aligned}
$$

where $c \in C(\mathfrak{g}, A)$ and $g_{1}, \ldots, g_{q+1} \in \mathfrak{g}$. We also set $C^{q}(\mathfrak{g}, A)=$ 0 for $q<0$ and $d_{q}=0$ for $q<0$. We can check that $d_{q+1} \circ d_{q}=0$ for all $q$ and therefore $\left\{C^{q}(\mathfrak{g}, A), d\right\}_{q \in \mathbb{Z}}$ is an algebraic complex. This complex is denoted by $C^{\bullet}(\mathfrak{g}, A)$, while $H^{q}(\mathfrak{g}, A)$ denotes the $q$-cohomology space of the algebra $\mathfrak{g}$ with coefficients in $A$. If $A$ is a trivial $\mathfrak{g}$-module, then the second sum of in the right-hand side of formula (22) vanishes and it may be ignored. If $A$ is a field, the notations $C^{q}(\mathfrak{g}, A)$, $H^{q}(\mathfrak{g}, A)$ are abbreviated to $C^{q}(\mathfrak{g}), H^{q}(\mathfrak{g})$.

2.2.2. Algebraic Interpretations of Cohomology. A derivation $\delta$ of the Lie algebra $\mathfrak{g}$ is a linear map $\delta: \mathfrak{g} \rightarrow \mathfrak{g}$ such that $\delta([x, y])=[\delta(x), y]+[x, \delta(y)]$. A derivation is inner if $\delta=$ $\delta_{x}(\cdot)=[x, \cdot]$, where $x \in \mathfrak{g}$ is a fixed element. Outer derivations are by definition elements of the quotient space of all derivations module the subspace of inner derivations. The proof of the following proposition is in [32], Chapter 1, Section 4.

Proposition 7. $H^{1}(\mathfrak{g}, \mathfrak{g})$ can be interpreted as the space of outer derivations of the algebra $\mathfrak{g}$.

Definition 8. A central extension of a Lie algebra $\mathfrak{g}$ by a vector space $\mathfrak{n}$ is a Lie algebra $\widetilde{\mathfrak{g}}$ whose underlying vector space $\widetilde{\mathfrak{g}}=\mathfrak{g} \oplus \mathfrak{n}$ is equipped with the following Lie bracket:

$$
[(X, u),(Y, v)]=([X, Y], c(X, Y))
$$

for some bilinear map $c: \mathfrak{g} \times \mathfrak{g} \rightarrow \mathfrak{n}$.

Note that $c$ depends only on $X$ and $Y$ but not on $u$ and $v$. This implies that $\mathfrak{n}$ is the center of the Lie algebra $\tilde{\mathfrak{g}}$.

The skew-symmetry, bilinearity, and the Jacoby identity on the Lie algebra $\widetilde{\mathfrak{g}}$ are equivalent to the antisymmetry, bilinearity, and the following 2-cocycle identity for the map $c$ :

$$
c([X, Y], Z)+c([Z, X], Y)+c([Y, Z], X)=0
$$

for any $X, Y, Z \in \mathfrak{g}$.

Two 2-cocycles $c$ on $\mathfrak{g}$ with values in $\mathfrak{n}$ differing by a 2 coboundary give rise to isomorphic central extensions of $\mathfrak{g}$. We have the following result, see [32, page 33].

Proposition 9. There is a one-to-one correspondence between equivalence classes of central extensions of $\mathfrak{g}$ by $\mathfrak{n}$ and elements of $H^{2}(\mathfrak{g}, \mathfrak{n})$.
The referee has pointed out that Proposition 9 combined with Proposition 10 below allows us to effectively calculate central extensions.

Proposition 10. Let $\mathfrak{g}$ be a Lie algebra. The space of onedimensional central extensions $H^{2}(\mathfrak{g}, \mathbb{C})$ is isomorphic to a subspace of the first cohomology space $H^{1}\left(\mathfrak{g}, \mathfrak{g}^{*}\right)$. Specifically, if we denote by $\bar{H}^{1}(\mathfrak{g})$ the subspace of $H^{1}\left(\mathfrak{g}, \mathfrak{g}^{*}\right)$ generated by cohomology classes of cocycles $\psi: \mathfrak{g} \rightarrow \mathfrak{g}^{*}$ such that

$$
\psi(x) \cdot x=0
$$

for $x \in \mathfrak{g}$, then $H^{2}(\mathfrak{g}, \mathbb{C}) \cong \bar{H}^{1}(\mathfrak{g})$.

This general proposition is due to Dzhumadil'daev, who used it in [33] (in the case of one-dimensional central extensions induced by fields of characteristic $p>0$ ) and in [34] (in the zero characteristic case) for the study of central extensions of Lie algebras of Cartan type; see, for example, [33-35]. Proposition 10 has the following corollary, also pointed out by the referee (see also $[23,33])$.

Corollary 11. If the Lie algebra $\mathfrak{g}$ admits an invariant, symmetric, and nondegenerate bilinear form $\langle$,$\rangle , then the space of one-$ dimensional central extensions $H^{2}(\mathfrak{g}, \mathbb{C})$ is isomorphic to the space of outer derivations $\delta: \mathfrak{g} \rightarrow \mathfrak{g}$ such that $\langle\delta(x), x\rangle=0$ for all $x \in \mathfrak{g}$.

Proof. The existence of $\langle$,$\rangle allows us to identify H^{1}(\mathfrak{g}, \mathfrak{g})$ with $H^{1}\left(\mathfrak{g}, \mathfrak{g}^{*}\right)$. Now, if we take $\psi$ so that the cohomology class of $\psi$ is in $\bar{H}^{1}(\mathfrak{g})$, we obtain a set of derivations of the form $\delta_{\psi}+[a, \cdot]$ for some $a \in \mathfrak{g}$. Then, invariance of $\langle$, $\rangle$ implies that $\psi \in$ $\bar{H}^{1}(\mathfrak{g})$ if and only if $\left\langle\delta_{\psi}(x), x\right\rangle=0$ for all $x \in \mathfrak{g}$.

2.3. Outer Derivations and Central Extensions of $\Psi D O$. We go back to the algebra $\Psi D O$ considered in Section 2.1. Following [16], we write formally the identity $\xi^{t}=e^{t \log \xi}$. This implies that

$$
\left.\frac{d}{d t}\right|_{t=0} \xi^{t}=\log \xi
$$

Hence, setting $\alpha=t$ in (8) and differentiating at $t=0$ using $\left.(d / d t)\right|_{t=0}\left(\begin{array}{c}t \\ j\end{array}\right)=(-1)^{j+1} / j$, we obtain

$$
\left[\log \xi, a \xi^{n}\right]=\sum_{j=1}^{\infty} \frac{(-1)^{j+1}}{j} \delta^{j}(a) \xi^{n-j} .
$$

It follows that if $P \in \Psi \mathrm{DO}$, then $[\log \xi, P]$ is also an element of $\Psi D O$, even though $\log \xi$ itself is not.

In the next proposition we will make use of the following combinatorial identity (see, for instance, [12]).

Lemma 12. Let $s \geq 1$ and $k \geq 0$ be integers and $\beta \in \mathbb{Z}$. Then

$$
\frac{(-1)^{s-1}}{s}\left(\begin{array}{c}
\beta-s \\
k
\end{array}\right)=\sum_{j=s}^{s+k} \frac{(-1)^{j-1}}{j}\left(\begin{array}{c}
\beta \\
s+k-j
\end{array}\right)\left(\begin{array}{c}
j \\
j-s
\end{array}\right) .
$$


Proposition 13 and Theorem 14 below were proved by Kravchenko and Khesin [16] in the case $\mathscr{A}=\operatorname{Diff}\left(S^{1}\right)$.

Proposition 13. [ $\log \xi, \cdot]$ defines a (resp., an outer) derivation of the associative (resp., Lie) algebra $\Psi D O$.

Proof. We note that the proposition does not follow from the fact that for any associative algebra the map $a \mapsto[a, \cdot]$ determines a derivation, since in our case $\log \xi$ is not an element of $\Psi D O$.

First of all, it is not difficult to see using (27) that [log $\xi, Q]$ belongs to $\Psi \mathrm{DO}$ for any $Q \in \Psi \mathrm{DO}$. Now, assuming that $[\log \xi, \cdot]$ is a derivation, it is trivial to prove that it is outer derivation of the Lie algebra $\Psi D O$ : if $[\log \xi, \cdot]=[A, \cdot]$ for some $A \in \Psi \mathrm{DO}$, then $\log \xi-A$ belongs to the center of $\Psi \mathrm{DO}$, and so $\log \xi \in \Psi D O$, a contradiction.

We show that $[\log \xi, \cdot]$ is a derivation. It is sufficient to prove that, for any $a, b \in A, m, n \in \mathbb{Z}$,

$$
\begin{aligned}
{\left[\log \xi, a \xi^{n} \circ b \xi^{m}\right]=} & {\left[\log \xi, a \xi^{n}\right] \circ b \xi^{m}+a \xi^{n} } \\
& \circ\left[\log \xi, b \xi^{m}\right] .
\end{aligned}
$$

Indeed, for the left side of (29) we have

$$
\begin{gathered}
{\left[\log \xi, a \xi^{n} \circ b \xi^{m}\right]=\left[\log \xi, \sum_{k=o}^{\infty}\left(\begin{array}{l}
n \\
k
\end{array}\right) a \delta^{j}(b) \xi^{n+m-k}\right]} \\
\quad=\sum_{k=o}^{\infty} \sum_{j=1}^{\infty}\left(\begin{array}{l}
n \\
k
\end{array}\right) \frac{(-1)^{j+1}}{j} \delta^{j}\left(a \delta^{k}(b)\right) \xi^{n+m-k-j} .
\end{gathered}
$$

On the other hand, for the right side of (29) we have

$$
\begin{aligned}
{\left[\log \xi, a \xi^{n}\right] \circ b \xi^{m}+a \xi^{n} \circ\left[\log \xi, b \xi^{m}\right] } \\
=\left(\sum_{j=0}^{\infty} \frac{(-1)^{j+1}}{j} \delta^{j}(a) \xi^{n-j}\right) b \xi^{m} \\
\quad+a \xi^{n}\left(\sum_{j=0}^{\infty} \frac{(-1)^{j+1}}{j} \delta^{j}(b) \xi^{m-j}\right) \\
=\sum_{j=1}^{\infty} \sum_{k=0}^{\infty}\left(\begin{array}{c}
n-j \\
k
\end{array}\right) \frac{(-1)^{j+1}}{j} \delta^{j}(a) \delta^{k}(b) \xi^{n+m-k-j} \\
\quad+\sum_{j=1}^{\infty} \sum_{k=0}^{\infty}\left(\begin{array}{c}
n \\
k
\end{array}\right) \frac{(-1)^{j+1}}{j} a \delta^{k}\left(\delta^{j}(b)\right) \xi^{n+m-k-j} \\
=\sum_{j=1}^{\infty} \sum_{k=0}^{\infty}\left(\begin{array}{c}
n-j \\
k
\end{array}\right) \frac{(-1)^{j+1}}{j} \delta^{j}(a) \delta^{k}(b) \xi^{n+m-k-j} \\
\quad+\sum_{j=1}^{\infty} \sum_{k=0}^{\infty}\left(\begin{array}{l}
n \\
k
\end{array}\right) \frac{(-1)^{j+1}}{j} a \delta^{k+j}(b) \xi^{n+m-k-j} .
\end{aligned}
$$

Now, for any integer $r \geqslant 1$, the coefficient of $\xi^{n+k-r}$ in (30) is

$$
\sum\left(\begin{array}{l}
n \\
k
\end{array}\right) \frac{(-1)^{j+1}}{j} \delta^{j}\left(a \delta^{k}(b)\right)
$$

where the summation is over all integers $j \geqslant 1, k \geqslant 0$ such that $j+k=r$. Using (9) we have

$$
\begin{aligned}
\sum \sum_{i=o}^{j}\left(\begin{array}{l}
n \\
k
\end{array}\right)\left(\begin{array}{l}
i \\
j
\end{array}\right) \frac{(-1)^{j+1}}{j} \delta^{j-i}(a) \delta^{i+k}(b) \\
=\sum \sum_{i=o}^{j-1}\left(\begin{array}{l}
n \\
k
\end{array}\right)\left(\begin{array}{l}
i \\
j
\end{array}\right) \frac{(-1)^{j+1}}{j} \delta^{j-i}(a) \delta^{i+k}(b) \\
+\sum\left(\begin{array}{l}
n \\
k
\end{array}\right) \frac{(-1)^{j+1}}{j} a \delta^{r}(b),
\end{aligned}
$$

where both summations are over all integers $j \geqslant 1, k \geqslant 0$ such that $j+k=r$. On the other hand, for $r \geqslant 1$, the coefficient $\xi^{n+k-r}$ of (31) is

$$
\begin{aligned}
& \sum\left(\begin{array}{c}
n-s \\
l
\end{array}\right) \frac{(-1)^{s+1}}{s} \delta^{s}(a) \delta^{l}(b) \\
& +\sum\left(\begin{array}{l}
n \\
l
\end{array}\right) \frac{(-1)^{s+1}}{s} a \delta^{r}(b),
\end{aligned}
$$

where the sum is over all integers $s \geqslant 1, l \geqslant 0$ such that $s+l=$ $r$. Therefore, (33) and (34) are equal if for fixed integers $s, l$ as above we have

$$
\sum\left(\begin{array}{l}
n \\
k
\end{array}\right)\left(\begin{array}{l}
j \\
i
\end{array}\right) \frac{(-1)^{j+1}}{j}=\left(\begin{array}{c}
n-s \\
l
\end{array}\right) \frac{(-1)^{s+1}}{s}
$$

where the sum is over all integers $j \geqslant 1, k \geqslant 0$, and $i \geqslant 0$ such that $i=j-s, i+k=l$. This amounts to showing that

$$
\sum_{j=s}^{l+s}\left(\begin{array}{c}
n \\
l+s-j
\end{array}\right)\left(\begin{array}{c}
j \\
j-s
\end{array}\right) \frac{(-1)^{j+1}}{j}=\left(\begin{array}{c}
n-s \\
l
\end{array}\right) \frac{(-1)^{s+1}}{s}
$$

and this is consequence of (28).

Theorem 14. The map $c: \Psi D O \times \Psi D O \rightarrow \mathbb{C}$ given by

$$
c(A, B)=\operatorname{res}([\log \xi, A] \circ B)
$$

defines a Lie algebra 2-cocycle on $\Psi D O$.

Proof. It is easy to see that $\operatorname{res}\left(\left[\log \xi, a \xi^{n}\right]\right)=0$ for $n \leq-1$, while for $n \geq 0$ we have

$$
\operatorname{res}\left(\left[\log \xi, a \xi^{n}\right]\right)=\tau\left(\frac{(-1)^{n}}{n+1} \delta^{n+1}(a)\right)=0,
$$

and so $\operatorname{res}([\log \xi, P])=0$ for all $P \in \Psi D O$. It follows that

$$
\begin{aligned}
c([B, A]) & =\operatorname{res}([\log \xi, B] A) \\
& =\operatorname{res}([\log \xi, B A]-B[\log \xi, A]) \\
& =-c(A, B),
\end{aligned}
$$

and so $c$ is skew-symmetric. It remains to prove the cocycle identity (24). This a direct calculation using Corollary 5 and the fact that $[\log \xi, \cdot]$ is a Lie algebra derivation. 
Remark 15. In the case of the Lie algebra of pseudodifferential symbols on $S^{1}$, the restriction of the cocycle (37) to the subalgebra of vector fields on $S^{1}$ is the Gelfand-Fuchs cocycle

$$
c(f(x) \partial, g(x) \partial)=\frac{1}{6} \int f^{\prime}(x) g^{\prime \prime}(x) d x ;
$$

see, for instance, [17, Prop. 4.12]. This cocycle is nontrivial; see [3], and therefore cocycle (37) is nontrivial [16]. It is also known (see, for instance, [17]) that the restriction of 2-cocycle (37) to the subalgebra of differential operator $\mathrm{DO}\left(S^{1}\right) \subset$ $\Psi \mathrm{DO}\left(S^{1}\right)$ is a multiple of the Kac-Peterson cocycle [6]:

$$
c\left(f \partial^{n}, g \partial^{m}\right)=\frac{n ! m !}{(m+n+1) !} \int_{S^{1}} f^{(m+1)} g^{(n)} d \theta .
$$

Interestingly, the Lie algebra $\mathrm{DO}\left(S^{1}\right)$ has exactly one central extension [36] but $\Psi \mathrm{DO}\left(S^{1}\right)$ has two independent central extensions $[17,37]$ : in addition to (37), the following expression defines a nontrivial cocycle:

$$
\widetilde{c}(A, B)=\int_{S^{1}}([x, A] \circ B) .
$$

We will reprove this result as a corollary of our study of central extensions of Lie algebras of formal pseudodifferential operators in several variables; see Lemma 33 in Section 3.4.

Remark 16. Let $\Sigma$ be a compact Riemann surface and let $\mathscr{M}$ be the space of meromorphic functions on $\Sigma$. Fix a meromorphic vector field $v$ on $\Sigma$ and denote by $D_{v}$ the operator of Lie derivative $L_{v}$ along the field $v$ : locally, if $v(x)=f(x) \partial / \partial x$, and $g \in \mathscr{M}$, then $D_{v}(g)=L_{v}(g(x))=f(x) g^{\prime}(x)$. The associative algebra of meromorphic pseudodifferential symbols is (see [4])

$$
M \Psi D O=\left\{\sum_{i=-\infty}^{n} a_{i} D_{v}{ }^{i} \mid a_{i} \in \mathscr{M}\right\},
$$

with multiplication defined as in (7). We consider the Lie algebra structure of $M \Psi D O$ and the residue map $\operatorname{res}_{D}\left(\sum_{i=-\infty}^{n} a_{i} D_{v}^{i}\right)=a_{-1} D_{v}^{-1}$, where $D_{v}^{-1}$ is understood as a meromorphic differential on $\Sigma$. We further define the trace associated to the point $P \in \Sigma$ by $\operatorname{Tr} A=\operatorname{res}_{P}\left(\operatorname{res}_{D}(A)\right)$. Then as a consequence of Theorem 14 we have a nontrivial 2-cocycle on $M \Psi D O$ given by $c_{v}(A, B)=\operatorname{Tr}\left(\left[\log D_{v}, A\right] B\right)$. This cocycle first appeared in [4].

2.4. A Hierarchy of Centrally Extended Lie Algebras. YDO is not unique in admitting nontrivial central extensions. In fact, a whole hierarchy of Lie algebras does. This fact was first observed by Khesin, [18], in the case $\mathscr{A}=\operatorname{Diff}\left(S^{1}\right)$.

For any positive integer $m$, we let $\Psi \mathrm{DO}_{m}$ be the subalgebra of $\Psi D O$ consisting of differential operators of the form $\sum_{i=m}^{M} a_{i} \xi^{i}$ for some nonnegative integer $M$.

Theorem 17. Suppose that the bilinear form defined in Corollary 5 is nondegenerate on $\Psi D O$. The restriction of the 2-cocycle $c(A, B)=\operatorname{res}([\log \xi, A] B)$ to $\Psi D O_{m}$ defines a nontrivial central extension of this subalgebra.
Proof. Using the bilinear form $\langle$,$\rangle defined in Corollary 5,$ we identify $\Psi \mathrm{DO}$ with the dual space $\Psi \mathrm{DO}^{*}$ and we have $\Psi \mathrm{DO}_{m}^{*} \simeq\left\{\sum_{i=-\infty}^{-m-1} a_{i} \xi^{i}: a_{i} \in \mathscr{A}\right\}$. If we assume that $c(A, B)$ is a coboundary, then (22) implies that for $A=\sum_{i=m}^{M} a_{i} \xi^{i}, B=$ $\sum_{i=m}^{M} b_{i} \xi^{i} \in \Psi \mathrm{DO}_{m}$, and a fixed $L=\sum_{i=-\infty}^{-m-1} l_{i} \xi^{i} \in \Psi \mathrm{DO}_{m}^{*}$ we have

$$
\begin{aligned}
& c(A, B)=\operatorname{res}([A, B] L)=\operatorname{res}\left(\left[\sum_{i=m}^{M} a_{i} \xi^{i}, \sum_{j=m}^{M} b_{j} \xi^{j}\right]\right. \\
& \left.\quad \cdot \sum_{k=-\infty}^{-m-1} l_{k} \xi^{k}\right) \\
& =\operatorname{res}\left(\sum _ { i , j , t , k , s , u = m , m , 0 , - \infty , 0 , 0 } \left((\text { coefficient }) \delta^{(s)}\left(a_{i}\right)\right.\right. \\
& \left.\left.\cdot \delta^{(t)}\left(b_{j}\right) \delta^{(u)}\left(l_{k}\right) \xi^{i+j+k-(t+u+s)}\right)\right) \\
& =\tau\left(\text { coefficient }_{-1}\right) .
\end{aligned}
$$

Then, the coefficient of $\xi^{-1}$ satisfies

$$
i+j+k-(t+u+s)=-1
$$

for $s, t, u \geq 0, k \leq-m-1$. On the other hand, we have that

$$
\begin{aligned}
& c(A, B)=\operatorname{res}([\log \xi, A] B)=\operatorname{res}\left(\left[\log \xi, \sum_{i=m}^{M} a_{i} \xi^{i}\right]\right. \\
& \left.\cdot \sum_{j=m}^{M} b_{j} \xi^{j}\right)=\operatorname{res}\left(\sum_{i, j, t, s=m, m, 0,0}(\text { coefficient }) \delta^{(s)}\left(a_{i}\right)\right. \\
& \left.\cdot \delta^{(t)}\left(b_{j}\right) \xi^{i+j-(s+t)}\right)=\tau\left(\text { coefficient }_{-1}\right) .
\end{aligned}
$$

Then, the coefficient of $\xi^{-1}$ satisfies

$$
i+j-(s+t)=-1
$$

comparing (45) and (47) for any $A, B$, and we find $k=u$, and this contradicts the condition for $k$ and $u$ appearing after (45).

We note that in this case we cannot use Corollary 11 to prove that the 2-cocycle $c$ determines a nontrivial central extension because $[\log \xi, \cdot]$ is not a derivation on $\Psi \mathrm{DO}_{m}$.

2.5. The Algebra of Twisted Pseudodifferential Symbols in One Variable. We consider the algebra of twisted pseudodifferential symbols and its corresponding logarithmic cocycle following [12]. Particular examples have appeared much earlier; see, for instance, $[11,19,20]$. 
Definition 18. Let $\sigma: \mathscr{A} \rightarrow \mathscr{A}$ be an automorphism of fixed algebra $\mathscr{A}$, and let $a, b \in \mathscr{A}$.

(1) A $\sigma$-derivation on $\mathscr{A}$ is a linear map $\delta$ such that $\delta(a b)=\delta(a) b+\sigma(a) \delta(b)$.

(2) A $\sigma$-trace on $\mathscr{A}$ is a linear map $\tau: \mathscr{A} \rightarrow \mathbb{C}$ such that $\tau(a b)=\tau(\sigma(b) a)$.

Given a triplet $(\mathscr{A}, \delta, \sigma)$ as above, the algebra of twisted formal pseudodifferential symbols $\Psi \mathrm{DO}_{\sigma}$ is the set of all formal Laurent series in $\xi$ with coefficients in $\mathscr{A}$ :

$$
\Psi \mathrm{DO}_{\sigma}=\left\{\sum_{i=-\infty}^{N} a_{i} \xi^{i}: N \in \mathbb{Z}, a_{n} \in \mathscr{A}\right\},
$$

equipped with a multiplication determined by the rules

$$
\begin{aligned}
\xi a & =\sigma(a) \xi+\delta(a), \\
\xi \xi^{-1} & =\xi^{-1} \xi=1 .
\end{aligned}
$$

For example, for each $n \geq 0$ we have

$$
\xi^{n} a=\sum_{i=0}^{n} P_{i, n}(\sigma, \delta)(a) \xi^{i}
$$

where $P_{i, n}(\sigma, \delta)$ is a noncommutative polynomial in $\sigma$ and $\delta$ with $\left(\begin{array}{c}n \\ i\end{array}\right)$ terms of total degree $n$ such that the degree of $\sigma$ is $i$. If $n=2$, for instance, we get $\xi^{2} a=\delta^{2}(a)+(\delta \sigma(a)+\sigma \delta(a)) \xi+$ $\sigma^{2}(a) \xi^{2}$. We extend (50) for $n<0$. We obtain

$$
\begin{aligned}
& \xi^{n} a=\sum_{i_{1}=0}^{\infty} \cdots \sum_{i_{n}=0}^{\infty}(-1)^{i_{1}+\cdots+i_{n}} \\
& \cdot \sigma^{-1}\left(\delta \sigma^{-1}\right)^{i_{n}} \cdots \sigma^{-1}\left(\delta \sigma^{-1}\right)^{i_{1}}(a) \xi^{n-i_{1}-\cdots-1_{n}},
\end{aligned}
$$

and it follows that if $A=\sum_{n=-\infty}^{N} a_{n} \xi^{n}$ and $B=\sum_{m=-\infty}^{M} b_{m} \xi^{m}$, then

$$
\begin{aligned}
A B & =\sum_{m=-\infty}^{M} \sum_{n<0} \sum_{i \geq 0}(-1)^{|i|} a_{n} \sigma^{-1}\left(\delta \sigma^{-1}\right)^{i-n} \\
& \cdots \sigma^{-1}\left(\delta \sigma^{-1}\right)^{i_{1}}\left(b_{m}\right) \xi^{m+n+|i|} \\
& +\sum_{m=-\infty}^{M} \sum_{n=0}^{N} \sum_{j=0}^{n} a_{n} P_{j, n}(\sigma, \delta)\left(b_{m}\right) \xi^{m+j},
\end{aligned}
$$

where $i=\left(i_{1}, \ldots, i_{n}\right)$ is an $n$-tuple of integers and $|i|=i_{1}+$ $\cdots+i_{n}$. The next proposition and theorem are proved in [12].

Proposition 19. Let $\mathscr{A}$ be algebra, $\sigma$ an automorphism of $\mathscr{A}, \tau$ a $\sigma$-trace on $\mathscr{A}$, and $\delta$ a $\sigma$-derivation on $\mathscr{A}$. If $\tau \circ \delta=0$, then for any $a, b \in \mathscr{A}$ and any $m$-tuple $i=\left(i_{1}, \ldots, i_{m}\right)$ of nonnegative integers, we have

$$
\begin{aligned}
& \tau\left(b \sigma^{-1}\left(\delta \sigma^{-1}\right)^{i_{1}} \cdots \sigma^{-1}\left(\delta \sigma^{-1}\right)^{i_{m}}(a)\right) \\
& \quad=(-1)^{i_{1}+\cdots+i_{m}} \tau\left(a \delta^{i_{m}} \sigma \delta^{i_{m-1}} \cdots \sigma \delta^{i_{1}}(b)\right) .
\end{aligned}
$$

Theorem 20. Let $\mathscr{A}$ be algebra, $\sigma$ an automorphism of $\mathscr{A}, \tau$ a $\sigma$-trace on $\mathscr{A}$, and $\delta$ a $\sigma$-derivation on $\mathscr{A}$. If $\tau \circ \delta=0$, then the linear functional res : $\Psi D O_{\sigma} \rightarrow \mathbb{C}$ defined by

$$
\operatorname{res}\left(\sum_{i=-\infty}^{n} a_{i} \xi^{i}\right)=\tau\left(a_{-1}\right)
$$

is a trace on $\Psi D O_{\sigma}$.

As pointed out in [12], if $\delta \circ \sigma=\sigma \circ \delta$, formulae (50) and (51) simplify to

$$
\xi^{n} a=\sum_{j=0}^{\infty}\left(\begin{array}{l}
n \\
j
\end{array}\right) \delta^{j}\left(\sigma^{n-j}(a)\right) \xi^{n-j} .
$$

For example, the twisted pseudodifferential operators considered in [11] satisfy (55). We introduce a twisted logarithmic cocycle assuming that $\delta$ and $\sigma$ commute. Let $\sigma_{t}$ be a 1parameter group of automorphisms of $\mathscr{A}$ with $\sigma_{1}=\sigma$. We formally replace the integer $n$ by $t \in \mathbb{R}$ in (55) and obtain

$$
\xi^{t} a-\sigma_{t}(a) \xi^{t}=\sum_{j=1}^{\infty}\left(\begin{array}{l}
t \\
j
\end{array}\right) \delta^{j}\left(\sigma_{t-j}\right) \xi^{t-j}
$$

Taking derivatives with respect to $t$ at $t=0$, as in Section 2.3 we obtain the commutation relation

$$
[\log \xi, a]=\left.\frac{d}{d t}\right|_{t=0} \sigma_{t}(a)+\sum_{j=1}^{\infty} \frac{(-1)^{j-1}}{j} \sigma^{-j} \delta^{j}(a) \xi^{-j}
$$

We note that $[\log \xi, \xi]=0$. The following two results are also proven in [12].

Proposition 21. The map $[\log \xi, \cdot]: \Psi D O_{\sigma} \rightarrow \Psi D O_{\sigma}$ defined by

$$
\begin{aligned}
{\left[\log \xi, a \xi^{n}\right]=} & \left.\frac{d}{d t}\right|_{t=0} \sigma_{t}(a) \xi^{n} \\
& +\sum_{j=1}^{\infty} \frac{(-1)^{j-1}}{j} \sigma^{-j} \delta^{j}(a) \xi^{-j}
\end{aligned}
$$

is a derivation.

Theorem 22. The 2-cochain $c(A, B)=\operatorname{res}([\log \xi, A] B)$ is a Lie algebra 2-cocycle.

Now we go beyond [12]. The algebra $\Psi_{\sigma}$ has a direct sum decomposition as a vector space,

$$
\Psi \mathrm{DO}_{\sigma}=\mathrm{DO}_{\sigma} \oplus \mathrm{INT}_{\sigma},
$$

where

$$
\begin{aligned}
& \mathrm{DO}_{\sigma}=\left\{\sum_{i=0}^{n} a_{i} \xi^{i} \mid a_{i} \in \mathscr{A}\right\}, \\
& \mathrm{INT}_{\sigma}=\left\{\sum_{i=-\infty}^{-1} a_{i} \xi^{i} \mid a_{i} \in \mathscr{A}\right\} .
\end{aligned}
$$


Moreover, we can prove a result analogous to Proposition 6, and we can also produce hierarchies of centrally extended algebras of twisted pseudodifferential symbols as in Theorem 17.

Proposition 23. $\operatorname{res}([A, B])=0$ for all $A, B \in \Psi D O_{\sigma}$. This implies that the bilinear form $\langle A, B\rangle_{\sigma}=\operatorname{res}(A B)$ is invariant; that is, it satisfies $\langle[A, B], C\rangle_{\sigma}=\langle A,[B, C]\rangle_{\sigma}$. Also, the subalgebras $D_{\sigma}$ and $I N T_{\sigma}$ are isotropic subspaces of $\Psi D O$; that is, the restrictions of the form $\langle,\rangle_{\sigma}$ to both $D_{\sigma}$ and $I N T_{\sigma}$ vanish.

Theorem 24. Suppose that the bilinear form defined in Proposition 23 is nondegenerate on $\Psi D O_{\sigma}$. The restriction of the 2-cocycle $c(A, B)=\operatorname{res}([\log \xi, A] B)$ to $\Psi D O_{\sigma, m}$ defines a nontrivial central extension of this subalgebra.

Proof. In addition to $\mathrm{DO}_{\sigma}$ and $\mathrm{INT}_{\sigma}$, we note that it is also possible to define subalgebras $\Psi \mathrm{DO}_{\sigma, m}$ of $\Psi \mathrm{DO}_{\sigma}$ by

$$
\Psi \mathrm{DO}_{\sigma, m}=\left\{\sum_{i=m}^{M} a_{i} \xi^{i} \mid a_{i} \in \mathscr{A}\right\} .
$$

The proof now follows along the lines of the demonstration of Theorem 17.

\section{The Algebra of Formal Pseudodifferential Symbols in Several Variables}

3.1. Preliminaries. Our notation mainly follows [23]. We fix $n \in \mathbb{Z}^{+}$and we set

$$
\begin{aligned}
\Gamma_{n} & =\left\{\alpha=\left(\alpha_{1}, \ldots, \alpha_{i}, \ldots, \alpha_{n}\right): \alpha_{i} \in \mathbb{Z}\right\} \\
\Gamma_{n}^{+} & =\left\{\alpha \in \Gamma_{n}: \alpha_{i} \in \mathbb{Z}^{+}, 1 \leq i \leq n\right\} ; \\
\varepsilon_{i} & =(0, \ldots, 0,1,0, \ldots, 0) ; \\
\vartheta & =\sum_{i=1}^{n} \varepsilon_{i} \in \Gamma_{n} ; \\
\alpha ! & =\prod_{i=1}^{n} \alpha_{i} !, \quad \alpha_{i} \in \Gamma_{n}^{+} .
\end{aligned}
$$

Also, $\xi^{\alpha}=\xi_{1}^{\alpha_{1}} \cdots \xi_{n}^{\alpha_{n}}, \delta^{\gamma}(b)=\delta_{1}^{\gamma_{1}} \circ \cdots \circ \delta_{n}^{\gamma_{n}}(b)$ for linear maps $\delta_{1}, \ldots, \delta_{n}$, and

$$
\left(\begin{array}{l}
\alpha \\
\gamma
\end{array}\right)=\prod_{i}\left(\begin{array}{l}
\alpha_{i} \\
\gamma_{i}
\end{array}\right)
$$

in which the binomial coefficient is defined as in Section 2.

Let $\mathscr{A}$ be an algebra on $\mathbb{C}$ and let $\delta_{i}$, with $i=1, \ldots, n$, be (commuting) derivations on $\mathscr{A}$. The algebra of formal differential symbols in several variables $\mathrm{DO}_{n}$ is, by definition, the algebra generated by $\mathscr{A}$ and symbols $\xi_{i}$ with the relations

$$
\xi_{i} a=a \xi_{i}+\delta_{i}(a)
$$

for all $a \in \mathscr{A}$ and $i=1, \ldots, n$. Elements of $\mathrm{DO}_{n}$ are of the form $A=\sum_{\alpha \in \Gamma_{n}^{+}} a_{\alpha} \xi^{\alpha}$. Using (64), we can prove that

$$
a \xi^{\alpha} \cdot b \xi^{\beta}=\sum_{\gamma \in \Gamma_{n}^{+}}\left(\begin{array}{l}
\alpha \\
\gamma
\end{array}\right) a \delta^{\gamma}(b) \xi^{\alpha+\beta-\gamma} .
$$

We extend the algebra $\mathrm{DO}_{n}$ to the algebra $\Psi_{n} \mathrm{DO}$ of formal pseudodifferential operators by introducing differentiations with negative exponents via

$$
\xi_{i}^{-1} a=\sum_{j \geq 0}(-1)^{j} \delta_{i}^{j}(a) \xi_{i}^{-1-j},
$$

and we define a structure of Lie algebra on $\Psi_{n}$ DO by the usual commutator

$$
\begin{aligned}
& {[A, B]=A B-B A} \\
& =\sum_{\gamma \in \Gamma_{n}^{+}}\left(\frac{1}{\gamma^{\prime}}\right)\left(\frac{\partial^{\gamma}}{\partial \xi^{\gamma}}(A) \delta^{\gamma}(B)-\frac{\partial^{\gamma}}{\partial \xi^{\gamma}}(B) \delta^{\gamma}(A)\right),
\end{aligned}
$$

where $\partial^{\gamma} / \partial \xi^{\gamma}$ and $\delta^{\gamma}$ are determined by linearity and the rules

$$
\begin{aligned}
\frac{\partial^{\gamma}}{\partial \xi^{\gamma}}\left(a \xi^{\alpha}\right) & =\gamma !\left(\begin{array}{l}
\alpha \\
\gamma
\end{array}\right) a \xi^{\alpha-\gamma}, \\
\delta^{\gamma}\left(a \xi^{\alpha}\right) & =\delta^{\gamma}(a) \xi^{\alpha} .
\end{aligned}
$$

3.2. The Logarithm of a Symbol and the Logarithmic 2-Cocycle. We define $\log \xi_{i}$ for all $i=1, \ldots, n$. As in Section 2, the action of $\log \xi_{i}$ on the algebra $\Psi_{n} \mathrm{DO}$ is via the commutator $\left[\log \xi_{i}, \cdot\right]$ considered in (67):

$$
\begin{aligned}
{\left[\log \xi_{i}, X\right] } & =\sum_{\gamma \in \Gamma_{n}^{+}} \frac{1}{\gamma !} \frac{\partial^{\gamma}}{\partial \xi^{\gamma}}\left(\log \xi_{i}\right) \delta^{\gamma}(X) \\
& =\sum_{\gamma_{i} \geq 0} \frac{1}{\gamma_{i} !} \frac{\partial_{i}^{\gamma}}{\partial \xi_{i}^{\gamma_{i}}}\left(\log \xi_{i}\right) \delta^{\gamma_{i}}(X)
\end{aligned}
$$

for all $X \in \Psi \mathrm{DO}_{n}$.

Proposition 25. The linear operator $\left[\log \xi_{i}, \cdot\right]$ defines a derivation of the (both associative and Lie) algebra $\Psi_{n} D O$ for all $i=1, \ldots, n$. The Lie algebra derivation $\left[\log \xi_{i}, \cdot\right]$ is outer.

Proof. One verifies, as in Section 2, that for any two symbols $A$ and $B$ in $\Psi D O$

$$
\left[\log \xi_{i}, A B\right]=\left[\log \xi_{i}, A\right] B+A\left[\log \xi_{i}, B\right],
$$

so that $\left[\log \xi_{i}, \cdot\right]$ is a derivation of the associative algebra. Equation (67) implies that $\left[\log \xi_{i}, \cdot\right]$ is also a derivation of the Lie algebra structure. That $\left[\log \xi_{i}, \cdot\right]$ is an outer derivation is proven as in Proposition 13.

Let $\tau$ be a $\delta_{i}$-invariant trace, $i=1, \ldots, n$, so that $\tau\left(\delta_{i}(a)\right)=$ 0 for all $a \in \mathscr{A}$. Then, as in Section 2, we have

$$
\tau\left(a \delta^{\gamma}(b)\right)=(-1)^{\gamma} \tau\left(b \delta^{\gamma}(a)\right) .
$$


Proposition 26. Let $\mathscr{A}$ be an algebra, $\delta_{i}$ derivations on $\mathscr{A}$, and $\tau$ a $\delta_{i}$-invariant trace, $i=1, \ldots, n$. Then, the linear functional res $: \Psi_{n} D O \rightarrow \mathbb{C} \Psi_{n} D O \rightarrow \mathbb{C}$ defined by

$$
\operatorname{res}\left(\sum_{\alpha \in \Gamma_{n}} a_{\alpha} \xi^{\alpha}\right)=\tau\left(a_{-\vartheta}\right)
$$

is a trace on $\Psi_{n} D O$.

Proof. It suffices to prove that, for any $a, b \in \mathscr{A}$ and $\alpha, \beta \in \Gamma_{n}$,

$$
\operatorname{res}\left(a \xi^{\alpha} b \xi^{\beta}\right)=\operatorname{res}\left(b \xi^{\beta} a \xi^{\alpha}\right) \text {. }
$$

Let $\alpha, \beta \in \Gamma_{n}$ such that for some $\alpha_{i}, \beta_{j}$, we have $\alpha_{i}, \beta_{j} \geq 0$ or $\alpha_{i}, \beta_{j}<0$; then (73) holds by Proposition 3. Thus, without loss of generality, we assume that, for all $i, \alpha_{i} \geq 0$ and $\beta_{i}<0$. If $\alpha_{i}+\beta_{i}<-1$, then using Proposition 3 we have (73) again. Now suppose that $\alpha_{i}+\beta_{i} \geq-1$ and let $k_{i}=\alpha_{i}+\beta_{i}$. We have

$$
\operatorname{res}\left(a \xi^{\alpha} b \xi^{\beta}\right)=\left(\begin{array}{c}
\alpha \\
k+\vartheta
\end{array}\right) \tau\left(a \delta^{k+\vartheta}\right) \text {. }
$$

On the other hand, applying (12) $n$ times and using (71), we obtain

$$
\begin{aligned}
\operatorname{res} & \left(b \xi^{\beta} a \xi^{\alpha}\right)=\tau\left(\left(\begin{array}{c}
\beta \\
k+\vartheta
\end{array}\right) b \delta^{k+\vartheta}(a)\right) \\
& =\left(\begin{array}{l}
k-\alpha \\
k+\vartheta
\end{array}\right) \tau\left(b \delta^{k+\vartheta}(a)\right) \\
& =(-1)^{k+\vartheta}\left(\begin{array}{c}
\alpha \\
k+\vartheta
\end{array}\right) \tau\left(b \delta^{k+\vartheta}(a)\right) \\
& =(-1)^{k+\vartheta}(-1)^{k+\vartheta}\left(\begin{array}{c}
\alpha \\
k+\vartheta
\end{array}\right) \tau\left(a \delta^{k+\vartheta}(b)\right) \\
& =\left(\begin{array}{c}
\alpha \\
k+\vartheta
\end{array}\right) \tau\left(a \delta^{k+\vartheta}(b)\right) .
\end{aligned}
$$

As in Section 2.1, we define the bilinear form $\langle A, B\rangle=$ $\operatorname{res}(A B)$ for $A, B \in \Psi_{n}$ DO. We can prove that it is symmetric and invariant, and we will assume hereafter that it is nondegenerate. Examples of nondegenerate bilinear forms as above appear in $[22,23]$ for special choices of algebras $\mathscr{A}$.

Theorem 27. The 2-cochains $c_{i}(A, B)=\operatorname{res}\left(\left[\log \xi_{i}, A\right] B\right), i=$ $1, \ldots, n$, are Lie algebra 2-cocycles of $\Psi_{n} D O$.

Proof. Skew-symmetry is proven as in Theorem 14. Also, as before, a straightforward computation using that $\left[\log \xi_{i}, \cdot\right]$ is a Lie algebra derivation yields $c_{i}([A, B], C)+c_{i}([C, A], B)+$ $c_{i}([B, C], A)=0$.

3.3. Hierarchies of Centrally Extended Algebras of Pseudodifferential Symbols in Several Variables. We present two examples of hierarchies of subalgebras of $\Psi_{n} \mathrm{DO}$ and we prove that they admit nontrivial central extensions. $n$, and

For our first example, we set $\alpha_{i}=m>0$ for fixed $1 \leq i \leq$

$$
\begin{aligned}
& \Psi_{n} \mathrm{DO}_{\alpha_{i}}=\left\{\sum_{\beta \in \Gamma_{n}} b_{\beta} \xi^{\beta}=\sum_{\left(\beta_{1}, \ldots, \beta_{n}\right) \in \Gamma_{n}} a_{\beta} \xi_{1}^{\beta_{1}} \cdots \xi_{n}^{\beta_{n}}\right. \\
& \left.\in \Psi_{n} \mathrm{DO}: \beta_{i} \geq \alpha_{i}\right\} .
\end{aligned}
$$

Theorem 28. The cocycle $c_{i}$ defined by $\log \xi_{i}$ is a nontrivial cocycle for the subalgebra $\Psi_{n} D O_{\alpha_{i}}$. Hence, it defines a nontrivial central extension of $\Psi_{n} D O_{\alpha_{i}}$.

Proof. We identify $\Psi_{n} \mathrm{DO}^{*}$ with $\Psi_{n} \mathrm{DO}$ making use of the nondegenerate bilinear form $\langle$,$\rangle . We also make the identi-$ fication $\Psi_{n} \mathrm{DO}_{\alpha_{i}}^{*} \simeq\left\{\sum_{\theta \in \Gamma_{n}} l_{\theta} \xi^{\theta}: \theta_{i} \leq-\alpha_{i}-1\right\}$. Let $A, B \in$ $\Psi_{n} \mathrm{DO}_{\alpha_{i}}$ and assume that $c_{i}$ is a coboundary. Then there exists $L \in \Psi_{n} \mathrm{DO}_{\alpha_{i}}^{*}$ such that for $A, B \in \Psi_{n} \mathrm{DO}_{\alpha_{i}}$ we have

$$
\begin{aligned}
& c(A, B)=\operatorname{res}([A, B] L) \\
& =\operatorname{res}\left(\left[\sum_{\alpha \in \Gamma_{n}} a_{\alpha} \xi^{\alpha}, \sum_{\beta \in \Gamma_{n}} b_{\beta} \xi^{\beta}\right] \sum_{\substack{\theta \in \Gamma_{n} \\
\theta_{i} \leq-\alpha_{i}-1}} l_{\theta} \xi^{\theta}\right) \\
& =\operatorname{res}\left(\sum \left((\text { coefficient }) \delta^{(\varphi)}\left(a_{\alpha}\right) \delta^{(\nu)}\left(b_{\beta}\right) \delta^{(\mu)}\left(l_{\theta}\right)\right.\right. \\
& \left.\left.\cdot \xi^{\alpha+\beta+\theta-(\nu+\mu+\varphi)}\right)\right)=\tau(\text { coefficient }-\vartheta) .
\end{aligned}
$$

The coefficient of $\xi^{-9}$ verifies that

$$
\alpha_{i}+\beta_{i}+\theta_{i}+\left(\nu_{i}+\mu_{i}+\varphi_{i}\right)=-1
$$

on the $i$ th position. On the other hand, we have that

$$
\begin{aligned}
& c(A, B)=\operatorname{res}\left(\left[\log \xi_{i}, A\right] B\right) \\
& =\operatorname{res}\left(\left[\log \xi_{i}, \sum_{\alpha \in \Gamma_{n}} a_{\alpha} \xi^{\alpha}\right] \sum_{\beta \in \Gamma_{n}} b_{\beta} \xi^{\beta}\right) \\
& =\operatorname{res}\left(\sum \text { (coefficient) } \delta_{i}^{\left(\psi_{i}\right)}\left(a_{\alpha}\right) \delta^{\left(\tau_{i}\right)}\left(b_{\beta}\right) \xi^{\alpha+\beta-\left(\psi_{i}+\tau\right)}\right) \\
& =\tau\left(\text { coefficient }_{-\vartheta}\right) .
\end{aligned}
$$

The coefficient of $\xi^{-9}$ satisfies that

$$
\alpha_{i}+\beta_{i}+\left(\tau_{i}+\varphi_{i}\right)=-1
$$

on the $i$ th position. Comparing (78) with (80) for arbitrary $A, B$ we have that $\mu_{i}=\theta_{i}$, and this is a contradiction, because $\theta_{i} \leq-\alpha_{i}-1$ and $\mu_{i} \geq 0$.

For our second example, let us say that $\alpha \geq \beta$ if $\alpha_{i} \geq \beta_{i}$ for all $i$. Fix $\alpha \in \Gamma_{n}^{+}$, and define $\Psi_{n} \mathrm{DO}_{\alpha}$ as the subalgebra of $\Psi_{n} \mathrm{DO}$ with elements of the form $\sum_{\beta \in \Gamma_{n}} b_{\beta} \xi^{\beta}$ such that $\beta \geq \alpha$. 
Theorem 29. The cocycle generated by $\log \xi_{i}$ for each $i$ is a nontrivial cocycle in $\Psi_{n} D O_{\alpha}$. Hence, it defines a nontrivial central extension of $\Psi_{n} D O_{\alpha}$.

Proof. The proof is similar to the proof of Theorem 28. We see that if the cocycle generated by $\log \xi_{i}$ were trivial, then (78) and (80) would be true for all $n$ components, and we would have $\mu_{i}=\theta_{i}$ for all $i$, which is impossible.

3.4. The Dzhumadil'daev Classification Theorem. We sketch a new proof of the principal theorem of [23]. It is a new proof, in the sense that we argue by induction and we use tools from homological algebra to perform the inductive step. It is a sketch, in the sense that full homological details are left for the paper [24]. We continue using the notation introduced in Section 3.1.

Let $P_{n}^{+}=\left\{\sum_{\alpha} \lambda_{\alpha} x^{\alpha} \mid \alpha \in \Gamma_{n}^{+}\right\}$be the algebra of polynomials in variables $x_{1}, \ldots, x_{n}$, and let $P_{n}$ be the algebra of Laurent power series of the form $\sum_{\alpha \in \Gamma_{n}} \lambda_{\alpha} x^{\alpha}$ such that the number of $\alpha \in \Gamma_{n}^{+}$'s with nonzero $\lambda_{\alpha}$ is finite. The action of the derivation $\delta_{i}$ on this algebra is determined by $\delta_{i}\left(x^{\alpha}\right)=$ $\alpha_{i} x^{\alpha-\epsilon_{i}}$, with $\alpha \in \Gamma_{n}$.

Let $\mathcal{U}$ be another algebra with derivations $\widetilde{\delta}_{1}, \ldots, \widetilde{\delta}_{n}$. The tensor product $\mathcal{U} \otimes P_{n}$ becomes an associative algebra if we endow it with the multiplication rule

$$
(u \otimes f)(v \otimes g)=\sum_{\alpha \in \Gamma_{n}^{+}} \frac{1}{\alpha !}\left(u \tilde{\delta}^{\alpha}(v) \otimes \delta^{\alpha}(f) g\right) .
$$

Clearly this algebra contains $\mathcal{U} \otimes P_{n}^{+}$as a subalgebra and we have the following.

Proposition 30. There is an isomorphism between the associative algebra of formal pseudodifferential symbols $\Psi_{n} D O$ and $\mathcal{U} \otimes P_{n}$ determined by the correspondence

$$
\begin{aligned}
\Psi_{n} D O & \longrightarrow \mathcal{U} \otimes P_{n} \\
u \xi^{\alpha} & \longmapsto u \otimes x^{\alpha} .
\end{aligned}
$$

This isomorphism determines a Lie algebra isomorphism between $\Psi_{n} D O$ and $\mathcal{U} \otimes P_{n}$.

Now, following [23], we let $\mathscr{H}_{n}$ be the Lie algebra associated to $\mathscr{U} \otimes P_{n}$ with $\mathscr{U}=P_{n}$. Then, identifying $x_{+}^{\alpha} x_{-}^{\beta} \simeq x^{\alpha} \otimes x^{\beta}$, we see that an element of $\mathscr{H}_{n}$ is of the form

$$
\sum_{\alpha, \beta \in \Gamma_{n}} \lambda_{\alpha, \beta} x_{+}^{\alpha} x_{-}^{\beta}
$$

and that the Lie bracket on $\mathscr{H}_{n}$ is given by

$$
\left[x_{+}^{\alpha} x_{-}^{\beta}, x_{+}^{\bar{\alpha}} x_{-}^{\bar{\beta}}\right]=\sum_{\gamma \in \Gamma_{n}^{+}}\left(\frac{1}{\gamma !}\right)\left(\partial_{-} \wedge \partial_{+}\right)\left(x_{+}^{\alpha} x_{-}^{\beta}, x_{+}^{\bar{\alpha}} x_{-}^{\bar{\beta}}\right)
$$

where $\partial_{+}, \partial_{-}$are derivations acting on $\mathscr{H}_{n}$ as

$$
\begin{aligned}
& \partial_{-}^{\gamma}\left(x_{+}^{\alpha} x_{-}^{\beta}\right)=\gamma !\left(\begin{array}{l}
\beta \\
\gamma
\end{array}\right) x_{+}^{\alpha} x_{-}^{\beta-\gamma}, \\
& \partial_{+}^{\gamma}\left(x_{+}^{\alpha} x_{-}^{\beta}\right)=\gamma !\left(\begin{array}{l}
\alpha \\
\gamma
\end{array}\right) x_{+}^{\alpha-\gamma} x_{-}^{\beta},
\end{aligned}
$$

and $\partial_{-} \wedge \partial_{+}=\partial_{-} \otimes \partial_{+}-\partial_{+} \otimes \partial_{-}$.

Definition 31. We define a linear function $f_{0}$ on $\mathscr{H}_{n}$ via $f_{0}\left(x^{-\vartheta}\right)=1$ and $f_{0}\left(x^{\alpha}\right)=0$ if $\alpha \neq-\vartheta$.

It is not hard to check that $f_{0}$ is an outer derivation of the Lie algebra $\mathscr{H}_{n}$. The following result is the main theorem of this section.

Theorem 32. The first group of cohomology of the Lie algebra $\mathscr{H}_{n}$ with coefficients in $\mathscr{H}_{n}, H^{1}\left(\mathscr{H}_{n}, \mathscr{H}_{n}\right)$, is generated (as space of outer derivations, see Proposition 7) by $f_{0}$, $\left[\log x_{+i}, \cdot\right]$ and $\left[\log x_{-i}, \cdot\right], i=1, \ldots, n$.

Proof. We sketch an inductive proof of Theorem 32. We consider first the one variable case; that is, the basic elements of the Lie algebra are $x_{+}=x_{+1}$ and $x_{-}=x_{-1}$. We write $\mathscr{H}$ instead of $\mathscr{H}_{1}$. Proposition 25 implies that $\left[\log x_{+}, \cdot\right]$ and $\left[\log x_{-}, \cdot\right]$ are outer derivations, and it is easy to check that the derivations $\left[\log x_{+}, \cdot\right],\left[\log x_{-}, \cdot\right]$, and $f_{0}$ are linearly independent. Now we have the following.

Lemma 33. Let $D$ be a derivation on $\mathscr{H}$ with $D\left(x_{ \pm}\right) \neq 0$. Then, there is $\omega \in \mathscr{H}$ such that

$$
D=\lambda\left[\log x_{+}, \cdot\right]+\beta\left[\log x_{-}, \cdot\right]-[\omega, \cdot]
$$

for some $\lambda, \beta$.

Proof. We know that $[u, 1]=0$ and then $[D(1), u]=0$ for all $u \in \mathscr{H}$. This implies that $D(1) \in Z(\mathscr{H})=\mathbb{C}$, and thus we can write $D(1)=\lambda$. Now, let $D\left(x_{+}\right)=\sum d_{n, m}^{+} x_{+}^{n} x_{-}^{m}$ and $D\left(x_{-}\right)=\sum d_{n, m}^{-} x_{+}^{n} x_{-}^{m}$. We have

$$
\begin{gathered}
1=\left[x_{-}, x_{+}\right] \Longrightarrow \lambda=\left[D\left(x_{-}\right), x_{+}\right]+\left[x_{-}, D\left(x_{+}\right)\right] \\
\Longrightarrow \lambda=\sum d_{n, m}^{-}\left[x_{+}^{n} x_{-}^{m}, x_{+}\right]+\sum d_{n, m}^{+}\left[x_{-}, x_{+}^{n} x_{-}^{m}\right] \\
\Longrightarrow \lambda=\sum d_{n, m}^{-} m x_{+}^{n} x_{-}^{m-1}+\sum d_{n, m}^{+} n x_{+}^{n-1} x_{-}^{m} .
\end{gathered}
$$

Equation (87) implies that $d_{n, m}^{+}=0$ except when $n=0$. Thus $d_{0, m} \neq 0$, and so $D\left(x_{+}\right)=\sum d_{0, m}^{+} x_{-}^{m}$. Analogously, we have that $D\left(x_{-}\right)=\sum d_{n, 0}^{-} x_{+}^{n}$. Also, (87) implies that

$$
d_{n, m}^{-} m x_{+}^{n} x_{-}^{m-1}=-d_{n, m} n x_{+}^{n-1} x_{-}^{m}
$$

except when $n=-1, m=-1$, and $(n, m)=(0,0)$. We rewrite (88) as

$$
(m+1) d_{n, m+1}^{-}=-(n+1) d_{n+1, m} .
$$


Then

$$
\begin{aligned}
& D\left(x_{+}\right)=\sum_{m \neq 0,-1}-d_{0, m}^{-} x_{-}^{m}+d_{0,-1} x_{-}^{-1}+d_{0,0}^{+}, \\
& D\left(x_{-}\right)=\sum_{n \neq 0,-1} d_{n, 0}^{-} x_{+}^{n}+d_{-1,0} x_{+}^{-1}+d_{0,0}^{-} .
\end{aligned}
$$

Now we write

$$
\begin{aligned}
D\left(x_{+}\right) & =\lambda\left[\log x_{+}, x_{+}\right]+\beta\left[\log x_{-}, x_{+}\right]-\left[\omega, x_{+}\right] \\
& =\beta x_{-}^{-1}-\left[\omega, x_{+}\right]
\end{aligned}
$$

for some $\omega$ to be determined. Comparing (90) and (92), we have that $\beta=d_{0,-1}^{+}$and

$$
\begin{aligned}
{\left[\omega, x_{+}\right] } & =\sum_{m \neq 0,-1} d_{0, m}^{-} x_{-}^{m}+d_{0,0}^{+} \Longrightarrow \partial_{-}(\omega) \\
& =\sum_{m \neq 0,-1} d_{0, m}^{-} x_{-}^{m}+d_{0,0}^{+} \Longrightarrow \omega \\
& =\sum_{m \neq 0,-1} \frac{d_{0, m}^{-}}{m+1} x_{-}^{m+1}+d_{0,0}^{+} x_{-}+\tilde{\omega}
\end{aligned}
$$

with $\left[\widetilde{\omega}, x_{+}\right]=0$. On the other hand,

$$
\begin{aligned}
D\left(x_{-}\right) & =\lambda\left[\log x_{+}, x_{-}\right]+\beta\left[\log x_{-}, x_{-}\right]-\left[\omega, x_{-}\right] \\
& =\lambda x_{+}^{-1}-\left[\omega, x_{-}\right] .
\end{aligned}
$$

Comparing (91) and (94) and using (93), we have that $\lambda=d_{0,1}^{-}$ and

$$
\begin{aligned}
{\left[\omega, x_{-}\right] } & =-\sum_{n \neq 0,-1} d_{n, 0}^{-} x_{+}^{n}+-d_{0,0}^{+} \Longrightarrow \partial_{-}(\omega) \\
& =-\sum_{n \neq 0,-1} d_{n, 0}^{-} x_{+}^{n}-d_{0,0}^{+} \Longrightarrow \omega \\
& =-\sum_{n \neq 0,-1} \frac{d_{n, 0}^{-}}{n+1} x_{+}^{n+1}-d_{0,0}^{-} x_{+}+\widetilde{\widetilde{\omega}}
\end{aligned}
$$

with $\left[\tilde{\widetilde{\omega}}, x_{-}\right]=0$. Then, we can take $\omega$ as

$$
\begin{aligned}
\omega= & -\sum_{n \neq 0,-1} \frac{d_{n, 0}^{-}}{n+1} x_{+}^{n+1} \\
& +\sum_{m \neq 0,-1} \frac{d_{0, m}^{-}}{m+1} x_{-}^{m+1}-d_{0,0}^{-} x_{+}-d_{0,0}^{-} x_{-} .
\end{aligned}
$$

Now we need the following result, whose proof (see [24]) is similar in spirit to the one we just carried out.

Lemma 34. If $D$ is an outer derivation such that $D\left(x_{ \pm}\right)=0$, then $D$ is a scalar multiple of $f_{0}$.

Lemmas 33 and 34 imply that Theorem 32 holds in the case $n=1$. In order to perform the inductive step we use an appropriate version of the Künneth formula. Again we refer to [24].
We use Theorem 32 to classify central extensions of $\mathscr{H}_{n}$ : Theorem 1 of [23] tells us that $\mathscr{H}_{n}$ can be equipped with the bilinear form $\langle u, v\rangle=\operatorname{res}(u v)=(u v)_{-9}$ and that this form is symmetric, nondegenerate, and invariant. Then, reasoning as in Theorem 27 we obtain that $c_{i}=\operatorname{res}\left(\left[\log \xi_{ \pm i}, A\right] B\right)$ and $c_{0}=\operatorname{res}\left(\left[f_{0}, A\right] B\right)$ are Lie algebra cocycles of $\mathscr{H}_{n}$. Now we use the key Corollary 11: we have that $\left\langle f_{0}(A), A\right\rangle \neq 0$ for $A=x^{-9}$ and therefore $f_{0}$ does not determine a central extension. On the other hand, in [23, page 135] the author shows that $\left\langle\left[\log \xi_{ \pm i}, A\right], A\right\rangle=0$ for all $A$. We conclude that the space of central extensions of $\mathscr{H}_{n}$ is of dimension $2 n$. The case $n=1$ is discussed from a geometric point of view in [17, Remark 4.16].

\section{Applications}

4.1. Manin Triples and Double Extensions of (Twisted) Pseudodifferential Symbols. Manin triples are ubiquitous in integrable systems; see, for instance, [17]. They were introduced by Drinfel'd in his seminal paper [15] on Hopf algebras and the quantum inverse scattering method.

Definition 35. Three Lie algebras $\overline{\mathfrak{g}}, \mathfrak{g}_{-}$, and $\mathfrak{g}_{+}$form a Manin triple if the following conditions are satisfied.

(1) The Lie algebras $\mathfrak{g}_{-}$and $\mathfrak{g}_{+}$are Lie subalgebras of $\overline{\mathfrak{g}}$ such that $\overline{\mathfrak{g}}=\mathfrak{g}_{-} \oplus \mathfrak{g}_{-}$as vector spaces.

(2) There exists a nondegenerate invariant bilinear form on $\overline{\mathfrak{g}}$ such that $\mathfrak{g}_{+}$and $\mathfrak{g}_{-}$are isotropic subspaces; that is, the restrictions of this form to both $\mathfrak{g}_{+}$and $\mathfrak{g}_{-}$ vanish.

For example, if we assume that the bilinear form $\langle A, B\rangle=$ $\operatorname{res}(A B)$ on $\Psi D O$ is nondegenerate, then Proposition 6 tells us that the algebras $\Psi D O, D O$, and INT form a Manin triple with respect to the bilinear form $\langle A, B\rangle$. In the same way, Proposition 23 implies that the algebras $\Psi \mathrm{DO}_{\sigma}, \mathrm{DO}_{\sigma}$, and $\mathrm{INT}_{\sigma}$ form a Manin triple with respect to the twisted bilinear form $\langle A, B\rangle_{\sigma}$.

Let us discuss the several variables case in some detail. We say that the order of the pseudodifferential operator $L=$ $\sum_{\alpha \in \Gamma_{n}} a_{\alpha} \xi^{\alpha}$ is $N$, if there is $\alpha=\left(\alpha_{1}, \ldots, \alpha_{n}\right)$ with $\alpha_{n}=N$ such that $a_{\alpha_{n}} \neq 0$ and for all $\beta=\left(\beta_{1}, \ldots, \beta_{n}\right)$ such that $\beta_{n}>N$ we have $a_{\beta}=0$. This definition is analogous to the notion of order used by Parshin in [22].

The Lie algebra $\Psi_{n}$ DO has two natural subalgebras:

$$
\begin{aligned}
& \mathrm{INT}_{n}=\left\{L \in \Psi_{n} \mathrm{DO} \mid \text { ord }(L)<0\right\}, \\
& \mathrm{DO}_{n}=\left\{L \in \Psi_{n} \mathrm{DO} \mid \text { ord }(L) \geq 0\right\},
\end{aligned}
$$

and clearly, as a vector space, $\Psi_{n}$ DO is a direct sum of these algebras: $\Psi_{n} \mathrm{DO}=\mathrm{INT}_{n} \oplus \mathrm{DO}_{n}$.

Proposition 36. If the bilinear form $\langle A, B\rangle=\operatorname{res}(A B)$ is nondegenerate, the algebras $\left(\Psi_{n} D O, I N T_{n}, D O_{n}\right)$ form a Manin triple with respect to $\langle$,$\rangle .$

Proof. We reason as in the proof of Proposition 6. 
We can also define order in $\Psi_{n} \mathrm{DO}$ by using a fixed coordinate $a_{i}$ instead of $\alpha_{N}, i \neq N$. Then, the Lie algebra $\Psi_{n} \mathrm{DO}$ has two natural subalgebras:

$$
\begin{aligned}
& \mathrm{INT}_{i}=\left\{\sum_{(\ldots,-\infty, \ldots) \in \Gamma_{n}}^{(\ldots,-1, \ldots)} a_{\alpha} \xi^{\alpha} \mid a_{\alpha} \in A\right\}, \\
& \mathrm{DO}_{i}=\left\{\sum_{(\ldots, 0, \ldots) \in \Gamma_{n}}^{(\ldots, n, \ldots)} a_{\alpha} \xi^{\alpha} \mid a_{\alpha} \in A\right\} .
\end{aligned}
$$

As before we have that as a vector space, $\Psi_{n} \mathrm{DO}=\mathrm{INT}_{i} \oplus \mathrm{DO}_{i}$.

Proposition 37. The algebras $\left(\Psi_{n} D O, I N T_{i}, D O_{i}\right)$ form a Manin triple with respect to the bilinear form $\langle A, B\rangle=\operatorname{res}(A B)$.

Proof. This is again analogous to the proof of Proposition 6.

Now we show how to construct further Manin triples with the help of central extensions using a general result by Bordemann, [25], which we now recall.

Let $\mathscr{A}$ a nonassociative algebra over a field $\mathbb{K}$ and assume that $f$ is a bilinear functional with the following properties:

(1) $f(a b, c)=f(a, b c)$ for all $a, b, c \in \mathscr{A}$.

(2) $f(a, b)=0$ for all $b \in \mathscr{A}$ implies $a=0$, and $f(a, b)=0$ for all $a \in \mathscr{A}$ implies $b=0$.

The pair $(\mathscr{A}, f)$ is called a pseudometrized algebra or if $f$ is symmetric, a metrized algebra. Bordemann's result is the following [25, Theorem 2.2].

Theorem 38. Let $(\mathscr{A}, f)$ be a metrized algebra over a field $\mathbb{K}$, and let $B$ be a Lie algebra over $\mathbb{K}$. Suppose that there is a Lie homomorphism $\phi: B \rightarrow \operatorname{Der}_{f}(\mathscr{A})$, where $\operatorname{Der}_{f}(\mathscr{A})$ denotes the space of all $f$-antisymmetric derivations of $\mathscr{A}$ (i.e., the derivations $d$ of $\mathscr{A}$ for which $f(d a, \widetilde{a})+f(a, d \widetilde{a})=0$ for all $a, \tilde{a} \in \mathscr{A}$ ).

Let $B^{*}$ denote the dual space of $B$. We define $w: \mathscr{A} \times \mathscr{A} \rightarrow$ $\mathscr{B}^{*}$ as the bilinear antisymmetric map

$$
(a, \widetilde{a}) \longmapsto(b \longmapsto f(\phi(b) a, \widetilde{a})),
$$

and for $b \in B$ and $\beta \in B^{*}$ we write $b \cdot \beta$ for the coadjoint representation; that is,

$$
(b \cdot \beta(\widetilde{b}))=-\beta(b \widetilde{b}) .
$$

We consider the vector space $\mathscr{A}_{B}=B \oplus A \oplus B^{*}$ and we define the following multiplication for $b, \widetilde{b} \in B, a, \widetilde{a} \in \mathscr{A}$, and $\beta, \widetilde{\beta} \in B^{*}$ :

$$
\begin{aligned}
& (b+a+\beta)(\widetilde{b}+\widetilde{a}+\widetilde{\beta}) \\
& =b \widetilde{b}+\phi(b) \tilde{a}-\phi(\widetilde{b}) a+a \widetilde{a}+w(a, \widetilde{a})+b \cdot \widetilde{\beta} \\
& \quad-\tilde{b} \cdot \beta .
\end{aligned}
$$

Moreover, we define the following symmetric bilinear form $f_{B}$ on $\mathscr{A}_{B}$ :

$$
f_{B}(b+a+\beta, \widetilde{b}+\widetilde{a}+\widetilde{\beta})=\beta(\widetilde{b})+\widetilde{\beta}(b)+f(a, \widetilde{a}) .
$$

The pair $\left(\mathscr{A}_{B}, f_{B}\right)$ is a metrized algebra over $\mathbb{K}$ called the double extension of $\mathscr{A}$ by $(\beta, \psi)$.

We sketch the proof of Theorem 38 in the case of interest for us. We assume that $\mathscr{A}$ is a Lie algebra and that $f(a, b)=$ $(a, b)$ is a bilineal symmetric form on $\mathscr{A}$ which is invariant and nondegenerate. Then, it is in fact easy to check that the bilineal form (102) is symmetric, nondegenerate and invariant. On the other hand, that (101) defines a Lie bracket on $\mathscr{A}_{B}$ follows from a straightforward computation using the identities

$$
\begin{aligned}
w\left(a,\left[a^{\prime}, \tilde{a}\right]\right) & =w\left(a^{\prime},[a, \tilde{a}]\right)-w\left(\tilde{a},\left[a, a^{\prime}\right]\right), \\
b \cdot w\left(a^{\prime}, \tilde{a}\right) & =w\left(a^{\prime}, \phi(b) \cdot \widetilde{a}\right)+w\left(\phi(b) \cdot a^{\prime}, \widetilde{a}\right)
\end{aligned}
$$

for $a, a^{\prime}, \widetilde{a} \in \mathscr{A}$ and $b \in B$.

As a first application we consider $\mathscr{A}=\Psi \mathrm{DO}, f\left(L_{1}, L_{2}\right)=$ $\left\langle L_{1}, L_{2}\right\rangle$ as in Section 2 , and $B=\{b[\log \xi, \cdot]: b \in \mathbb{R}\}, B^{*}=\mathbb{R}$, and we set $\phi: B \rightarrow \operatorname{Der}_{f}(\mathscr{A})$ as the inclusion map. Then, $w\left(L_{1}, L_{2}\right)=\left\langle\left[\log \xi, L_{1}\right], L_{2}\right\rangle$ and Theorem 38 gives us the double extension $\widetilde{\Psi D O}=B \oplus \Psi \mathrm{DO} \oplus B^{*}$ with bracket

$$
\begin{aligned}
& {\left[\left(b_{1}, L_{1}, \beta_{1}\right),\left(b_{2}, L_{2}, \beta_{2}\right)\right]=\left(0, b_{1}\left[\log \xi, L_{2}\right]\right.} \\
& \left.\quad-b_{2}\left[\log \xi, L_{1}\right]+\left[L_{1}, L_{2}\right],\left\langle\left[\log \xi, L_{1}\right], L_{2}\right\rangle\right)
\end{aligned}
$$

and invariant symmetric linear form

$$
\begin{aligned}
& \left\langle\left(b_{1}, L_{1}, \beta_{1}\right),\left(b_{2}, L_{2}, \beta_{2}\right)\right\rangle \\
& \quad=\beta_{1} b_{2}+\beta_{2} b_{1}+\operatorname{res}\left(L_{1} L_{2}\right) .
\end{aligned}
$$

This extension is certainly known; see [17]. In an analogous way we obtain a double extension $\widetilde{\mathrm{YDO}_{\sigma}}$ of the Lie algebra of twisted pseudodifferential operators.

Now, we note that the Lie algebra $\widetilde{\Psi D O}$ (and also $\widetilde{\Psi D O_{\sigma}}$ ) admits the (vector space) direct sum decomposition $\widetilde{\Psi D O}=$ $\widehat{\mathrm{DO}} \oplus \widetilde{\mathrm{INT}}$ (resp., $\widetilde{\Psi \mathrm{YO}_{\sigma}}=\widehat{\mathrm{DO}_{\sigma}} \oplus \widetilde{\mathrm{INT}_{\sigma}}$ ), in which

$$
\begin{aligned}
& \widehat{\mathrm{DO}}=\left\{\alpha c+\sum_{i=0}^{n} a_{i} \xi^{i} \mid a_{i} \in \mathscr{A}, \alpha \in \mathbb{R}\right\}, \\
& \widetilde{\mathrm{INT}}=\left\{\beta \log \xi+\sum_{i=-\infty}^{-1} a_{i} \xi^{i} \mid a_{i} \in \mathscr{A}, \beta \in \mathbb{R}\right\},
\end{aligned}
$$

and analogous definitions for the twisted case. We have the following.

Corollary 39. If the bilinear form (102) is nondegenerate, then

$$
\begin{gathered}
(\widetilde{\Psi D O}, \widehat{D O}, \overparen{I N T}), \\
\left(\widetilde{\Psi_{\sigma} D O}, \widehat{D O_{\sigma}}, \widetilde{I N T_{\sigma}}\right)
\end{gathered}
$$

are Manin triples. 
Now we consider the doubly extended algebra $\widetilde{\Psi_{n} \mathrm{DO}}$ of $\Psi_{n} \mathrm{DO}$, that is, the semidirect product of $\widehat{\Psi_{n} \mathrm{DO}}=\Psi_{n} \mathrm{DO} \oplus \mathbb{R} \cdot c_{i}$ and the space of derivations $\left\{\beta \log \xi_{i} \mid \beta \in \mathbb{R}\right\}$. We have the vector space decomposition

$$
\widetilde{\Psi_{n} \mathrm{DO}}=\widetilde{\mathrm{INT}}_{i} \oplus \widehat{\mathrm{DO}}_{i},
$$

where

$$
\begin{aligned}
& \widetilde{\mathrm{INT}}_{i}=\left\{\beta \log \xi_{i}+\sum_{(\ldots,-\infty, \ldots) \in \Gamma_{n}}^{(\ldots,-1, \ldots)} a_{\theta} \xi^{\theta} \mid a_{\theta} \in A\right\}, \\
& \widehat{\mathrm{DO}}_{i}=\left\{\alpha c_{i}+\sum_{(\ldots, 0, \ldots) \in \Gamma_{n}}^{(\ldots, n, \ldots)} a_{\theta} \xi^{\theta} \mid a_{\theta} \in A\right\} .
\end{aligned}
$$

Theorem 38 yields the corollary.

Corollary 40. The algebras $\left(\widetilde{\Psi_{n} D O}, \overparen{I N T_{i}}, \widehat{D O_{i}}\right)$ form a Manin triple with respect to the bilinear form $\left\langle A+b_{1} c_{i}+\right.$ $\left.\alpha_{1} \log \xi_{i}, B+b_{2} c_{i}+\alpha_{2} \log \xi_{i}\right\rangle=\operatorname{res}(A B)+b_{2} \alpha_{1}+b_{1} \alpha_{2}$ and Lie bracket $\left[\left(A, b_{1}, \alpha_{1}\right),\left(B, b_{2}, \alpha_{2}\right)\right]=\left([A, B]+\left[\alpha_{2} \log \xi_{i}, A\right]-\right.$ $\left.\left[\alpha_{1} \log \xi_{i}, B\right], c_{i}(A, B), 0\right)$.

Theorem 38 also allows us to define a double extension of $\overline{\Psi_{n} \mathrm{DO}}$ by considering the $n$-central extension $\mathbb{R} \cdot c_{1} \oplus$ $\cdots \mathbb{R} \cdot c_{n}$ and the $n$ symbols $\log \xi_{i}$. We have the vector space decomposition

$$
\begin{aligned}
\widetilde{\Psi_{n} \mathrm{DO}}= & \Psi_{n} \mathrm{DO} \oplus\left[\left(\mathbb{R} \cdot c_{1}\right) \oplus \cdots \oplus\left(\mathbb{R} \cdot c_{n}\right)\right] \\
& \oplus\left[\left(\mathbb{R} \cdot \log \xi_{1}\right) \oplus \cdots \oplus\left(\mathbb{R} \cdot \log \xi_{n}\right)\right],
\end{aligned}
$$

with Lie bracket given by

$$
\begin{aligned}
& {[(A, \alpha, \beta),(B, \bar{\alpha}, \bar{\beta})]=([A, B]+[\bar{\beta} \cdot \log \xi, A]} \\
& \quad-[\beta \cdot \log \xi, B], c(A, B), 0),
\end{aligned}
$$

and bilinear form

$$
\langle A, B\rangle=\operatorname{res}(A, B)+\alpha \cdot \bar{\beta}+\beta \cdot \bar{\alpha},
$$

where $\alpha, \beta, \bar{\alpha}$, and $\bar{\beta}$ denote $n$-tuples, $\log \xi=\left(\log \xi_{1}, \log \xi_{2}\right.$, $\left.\ldots, \log \xi_{n}\right), 0=(0, \ldots, 0) \in \mathbb{R}^{n}$, and $c(A, B)=\left(c_{1}(A, B)\right.$, $\left.\ldots, c_{n}(A, B)\right)$. The operation "." is the usual dot product in $\mathbb{R}^{n}$; for example, $\beta \cdot \log \xi=\beta_{1} \log \xi+\cdots+\beta_{n} \log \xi_{n}$.

4.2. Hierarchies of Differential Equations. In this last section we apply our work to the construction of hierarchies of partial differential equations. We begin with some standard facts on hamiltonian systems modelled on (dual spaces of) Lie algebras (see, for instance, [17] or the recent paper [38]).

Definition 41. If $\mathfrak{g}$ is a Lie algebra and $\mathfrak{g}^{*}$ is its dual space, the functional derivative of a function $F: \mathfrak{g}^{*} \rightarrow \mathbb{R}$ at $\mu \in \mathfrak{g}^{*}$ is the unique element $\delta F / \delta \mu$ of $\mathfrak{g}$ determined by

$$
\left\langle\nu, \frac{\delta F}{\delta \mu}\right\rangle=\left.\frac{d}{d \epsilon}\right|_{\epsilon=0} F(\mu+\epsilon \nu)
$$

for all $\nu \in \mathfrak{g}^{*}$, in which $\langle$,$\rangle denotes a natural paring between$ $\mathfrak{g}$ and $\mathfrak{g}^{*}$.
Definition 42. The Lie-Poisson bracket on the dual space $\mathfrak{g}^{*}$ is given by

$$
\{F, G\}(\mu)=\left\langle\mu,\left[\frac{\delta F}{\delta \mu}, \frac{\delta G}{\delta \mu}\right]\right\rangle,
$$

in which $F, G: \mathfrak{g}^{*} \rightarrow \mathbb{R}$ and $\mu \in \mathfrak{g}^{*}$.

The corresponding equations of motion for a Hamiltonian $H: \mathfrak{g}^{*} \rightarrow \mathbb{R}$ are

$$
\frac{d \mu}{d t}=\left\langle\mu,\left[, \frac{\delta H}{\delta \mu}\right]\right\rangle .
$$

Definition 43. Let $(\mathfrak{g},[]$,$) be a Lie algebra and R: \mathfrak{g} \rightarrow \mathfrak{g}$ a linear operator on $\mathfrak{g}$. We define a bilinear antisymmetric bracket $[,]_{R}$ on $\mathfrak{g}$ by

$$
[P, Q]_{R}=[R(P), Q]+[P, R(Q)] \quad Q, P \in \mathfrak{g} .
$$

The operator $R$ is called a classical $R$-matrix if the bracket $[,]_{R}$ satisfies the Jacobi identity.

If $\mathfrak{g}=\mathfrak{g}_{-} \oplus \mathfrak{g}_{+}$as vector space, we choose $R=(1 / 2)\left(\pi_{+}-\right.$ $\pi_{-}$), where $\pi_{ \pm}$are the projection operators on $\mathfrak{g}_{ \pm}$. We write $P_{ \pm}=\pi_{ \pm}(P)$ for $P \in \mathfrak{g}$. Then, $[P, Q]_{R}=\left[P_{+}, Q_{+}\right]-\left[P_{-}, Q_{-}\right]$and

$$
\{F, G\}_{R}=\left\langle\mu,\left[\frac{\delta F}{\delta \mu}, \frac{\delta G}{\delta \mu}\right]_{R}\right\rangle .
$$

If $F: \mathfrak{g}^{*} \rightarrow \mathbb{R}$ satisfies the infinitesimal $A d^{*}$-invariance condition

$$
\left\langle\mu,\left[\frac{\delta F}{\delta \mu}, \cdot\right]\right\rangle=0
$$

for all $\mu \in \mathfrak{g}^{*}$, bracket (117) becomes

$$
\{F, G\}_{R}=\left\langle\mu,\left[\left(\frac{\delta F}{\delta \mu}\right)_{+}, \frac{\delta G}{\delta \mu}\right]\right\rangle .
$$

It follows that if $H$ is a hamiltonian function on $\mathfrak{g}^{*}$ satisfying (118), the corresponding hamiltonian vector field is given by

$$
X_{H}(\mu) \cdot F=\left\langle\mu,\left[-\left(\frac{\delta H}{\delta \mu}\right)_{+}, \frac{\delta F}{\delta \mu}\right]\right\rangle
$$

and, if there exists a nondegenerate pairing $\langle$,$\rangle between \mathfrak{g}$ and $\mathfrak{g}^{*}$ such that the invariance condition

$$
\langle P[Q, R]\rangle=\langle[R, P], Q\rangle
$$

holds for $P, Q, R \in \mathfrak{g}$, then the corresponding equations of motion with respect to the Poisson structure (119) can be written as equations on $\mathfrak{g}$ as

$$
\frac{d P}{d t}=\left[\left(\frac{\delta H}{\delta \mu}\right)_{+}, P\right] .
$$


4.2.1. The KP Hierarchy. We apply the above remarks to the Lie algebra $\Psi D O$. Let us recall that we have a bilinear symmetric form on $\Psi D O$ which satisfies

$$
\langle[P, Q], S\rangle=\langle[S, P], Q\rangle \quad S, P, Q \in \Psi D O
$$

We assume that this form is nondegenerate. The decomposition $\Psi \mathrm{DO}=\mathrm{INT} \oplus \mathrm{DO}=\Psi \mathrm{DO}_{-} \oplus \Psi \mathrm{DO}_{+}$allows us to define a new structure of Lie algebra $[,]_{R}$ on $\Psi D O$ using $R$ matrices as above. The following proposition is standard; see, for instance, [5] or the recent work [38].

Proposition 44. Let $H_{k}(L)=\operatorname{res}\left(L^{k}\right), k=1,2, \ldots$ for $L \in$ IDO. Then,

$$
\frac{\delta H_{k}}{\delta L}=k L^{k-1}
$$

Moreover, for any functional $F$ on $\Psi D O^{*},\left\{H_{k}, F\right\}(L)=0$, and $\left\{H_{k}, H_{l}\right\}_{R}(L)=0$ for any $R$-matrix $R$.

Equation (122) gives us the standard Kadomtsev-Petviashvili hierarchy as a Hamiltonian equation.

Theorem 45. Let $H_{k}(L)=\operatorname{res}\left(L^{k}\right)$ for $L \in \Psi D O$. The corresponding Hamiltonian equation of motion with respect to $\{\cdot, \cdot\}_{R}$ is

$$
\frac{\partial L}{\partial t}=\left[\left(k L^{k-1}\right)_{+}, L\right]
$$

4.2.2. The Twisted KP Hierarchy. We consider (59) and Proposition 23. The Manin triple $\Psi \mathrm{DO}_{\sigma}, \mathrm{DO}_{\sigma}$, and $\mathrm{INT}_{\sigma}$ allows us to consider a new Lie bracket $[,]_{R}$ on $\Psi \mathrm{DO}_{\sigma}$ as in the previous subsection. Proposition 44 is also valid in the twisted context (see the proof appearing, for instance, in [38]), and therefore (122) yields the following result.

Theorem 46. Let $H_{k}(L)=\operatorname{res}\left(L^{k}\right)$ for $L \in \Psi D O_{\sigma}$. The corresponding Hamiltonian equation of motion with respect to $\{\cdot, \cdot\}_{R}$ is

$$
\frac{\partial L}{\partial t}=\left[\left(k L^{k-1}\right)_{+}, L\right]
$$

A special case of (126) appears in [11]; see (7.4) therein.

4.2.3. The $n$-KP Hierarchy. Now we consider Proposition 36. As before, the Manin triple $\Psi_{n} \mathrm{DO}, \mathrm{DO}_{n}$, and $\mathrm{INT}_{n}$ allows us to induce a new Lie algebra structure on $\Psi_{n} \mathrm{DO}$ with bracket $[,]_{R}$. Once more we set $H_{k}(L)=\operatorname{res}\left(L^{k}\right)$ for $L \in \Psi_{n} \mathrm{DO}$. Equation (122) yields the equation of motion

$$
\frac{\partial L}{\partial t}=\left[\left(k L^{k-1}\right)_{+}, L\right] \text {. }
$$

This is an $n$-variables generalization of KP of the kind considered by Parshin in [22].

4.2.4. Zero Curvature Equations. Now we add a central extension $c$ to $\Psi \mathrm{DO}$ and we study $\widehat{\Psi D O}=\Psi \mathrm{DO} \oplus \mathbb{R}$.
Definition 47. Let $(A, \alpha),(B, \beta) \in \widehat{\Psi D O}$. We define a bilinear form on $\widehat{\Psi D O}$ by

$$
\langle(A, \alpha),(B, \beta)\rangle=\langle A, B\rangle+\alpha \beta=\operatorname{res}(A B)+\alpha \beta .
$$

We use this bilinear form to identify the dual space of $\widehat{\Psi D O}$ with itself.

Proposition 48. Let $H:(\widehat{\Psi D O})^{*} \rightarrow \mathbb{R}$ be a Hamiltonian function. The Hamiltonian equations of motion with respect to the bracket (114) are

$$
\begin{aligned}
\left\langle\frac{\partial L}{\partial t}, Q\right\rangle & =\left\langle\left[\frac{\partial H}{\partial L}, L\right], Q\right\rangle+l c\left(Q, \frac{\delta H}{\delta L}\right), \\
\frac{d l}{d t} & =0
\end{aligned}
$$

for any $Q \in \Psi D O$, in which $(\widetilde{L}, \widetilde{l})=\langle(L, l),(\cdot, \cdot)\rangle \in(\widehat{\Psi D O})^{*}$.

Proof. The Hamiltonian equations of motion on $(\widehat{\Psi \mathrm{DO}})^{*}$ with Hamiltonian $H$ are

$$
\begin{aligned}
& \frac{d(\widetilde{L}, \tilde{l})}{d t} \cdot(Q, r)=\left\langle(\widetilde{L}, \tilde{l}),\left[(Q, r), \frac{\delta H}{\delta(\widetilde{L}, \widetilde{l})}\right]\right\rangle \\
& =\left\langle(\widetilde{L}, \tilde{l}),\left[(Q, r),\left(\frac{\delta H}{\delta \widetilde{L}}, \frac{\delta H}{\delta \widetilde{l}}\right)\right]\right\rangle \\
& =\left\langle(\widetilde{L}, \tilde{l}),\left(\left[Q, \frac{\delta H}{\delta \widetilde{L}}\right], c\left(Q, \frac{\delta H}{\delta \widetilde{L}}\right)\right)\right\rangle .
\end{aligned}
$$

If we set $(\widetilde{L}, \widetilde{l})=\langle(L, l),(\cdot, \cdot)\rangle$ for some $(L, l) \in \widehat{\Psi D O}$, the last equation becomes

$$
\begin{aligned}
& \left\langle\frac{\partial(L, l)}{\partial t},(Q, r)\right\rangle \\
& =\left\langle(L, l),\left(\left[Q, \frac{\delta H}{\delta L}\right], c\left(Q, \frac{\delta H}{\delta L}\right)\right)\right\rangle \\
& =\left\langle L,\left[Q, \frac{\delta H}{\delta L}\right]\right\rangle+l c\left(Q, \frac{\delta H}{\delta L}\right) .
\end{aligned}
$$

Using invariance of the bilinear form $\langle$,$\rangle , we write this$ equation as

$$
\left\langle\frac{\partial L}{\partial t}, Q\right\rangle+r \frac{\partial l}{\partial t}=\left\langle\left[\frac{\delta H}{\delta L}, L\right], Q\right\rangle+l c\left(Q, \frac{\delta H}{\delta L}\right) .
$$

Since this equation is valid for any $(Q, r)$, we conclude that $d l / d t=0$ and we obtain (129).

In the context of current algebras, (129) and (130) yield the zero curvature equations obtained by Reyman and SemenovTian-Shansky in [28]. 
Finally, we restrict our attention to the subalgebra Vect $=$ $\{a \xi: a \in \mathscr{A}\}$. In this case, we can compute the cocycle $c$ rather easily:

$$
\begin{aligned}
& \operatorname{res}([\log \xi, a \xi] b \xi)=\operatorname{res}(b \xi[\log \xi, a \xi]) \\
& \quad=\operatorname{res}\left(b \xi \sum_{j=1}^{\infty} \frac{(-1)^{j+1}}{j} \delta^{j}(a) \xi^{1-j}\right) \\
& =\operatorname{res}\left(\sum_{j=0}^{\infty} \frac{(-1)^{j+1}}{j}\left(b \delta^{j}(a) \xi^{2-j}+b \delta^{j+1}(a) \xi^{1-j}\right)\right) \\
& =\frac{(-1)^{3+1}}{3} b \delta^{3}(a)+\frac{(-1)^{2+1}}{2} b \delta^{3}(a) \\
& =-\frac{1}{6} \tau\left(b \delta^{3}(a)\right) .
\end{aligned}
$$

We consider the subalgebra Vect $\oplus \mathbb{R}$ of $\widehat{\Psi D O}$, and we equip it with the bilinear form

$$
\langle(a \xi, \alpha),(b \xi, \beta)\rangle=\tau(a b)+\alpha \beta .
$$

Then, if we set $(L, l)=(u \xi, l), Q=b \xi$, and $\delta H / \delta L=h(u) \xi$, and we use (129) and (130), which were derived without using the specific form of the inner product on $\widehat{\Psi D O}$, we obtain

$$
\begin{aligned}
\left\langle\frac{d u}{d t} \xi, b \xi\right\rangle= & \langle[h(u) \xi, u \xi], b \xi\rangle \\
& -\frac{1}{6} l \tau\left(b \delta^{3}(h(u))\right) ;
\end{aligned}
$$

that is, using that $\tau$ is nondegenerate,

$$
\frac{d u}{d t}=h(u) \delta(u)-u \delta(h(u))-\left(\frac{1}{6}\right) l \delta^{3}(h(u)),
$$

an abstract form of the ubiquitous Korteweg-de Vries equation.

\section{Conflict of Interests}

The authors declare that there is no conflict of interests regarding the publication of this paper.

\section{Acknowledgments}

Jarnishs Beltran's research has been partially supported by the Universidad del Desarrollo and the Project MECESUPUSACH PUC 0711. Enrique G. Reyes' research has been partially supported by the Project FONDECYT no. 1111042 and the USACH DICYT grant 041533RG.

\section{References}

[1] V. Ovsienko and C. Roger, "Deforming the Lie algebra of vector fields on $S^{1}$ inside the Lie algebra of pseudodifferential symbols on $S^{1}$, Translations of the American Mathematical Society, Series 2, vol. 194, pp. 211-226, 1999.
[2] L. Guieu and C. Roger, L’Algébre et le Groupe de Virasoro. Aspects géom Étriques et Algébriques, Généralisations, Les Publications CRM, Montreal, Canada, 2007.

[3] I. M. Gelfand and D. B. Fuchs, "Cohomology of the Lie algebra of the vector field on the circle," Functional Analysis and Its Applications, vol. 2, pp. 342-343, 1969.

[4] D. Donin and B. Khesin, "Pseudodifferential symbols on Riemann surfaces and Krichever-Novikov algebras," Communications in Mathematical Physics, vol. 272, no. 2, pp. 507-527, 2007.

[5] L. A. Dickey, Soliton Equations and Hamiltonian Systems, vol. 12 of Advanced Series in Mathematical Physics, World Scientific Publishing, River Edge, NJ, USA, 1991.

[6] V. G. Kac and D. H. Peterson, "Spin and wedge representations of infinite-dimensional Lie algebras and groups," Proceedings of the National Academy of Sciences of the United States of America, vol. 78, no. 6, part 1, pp. 3308-3312, 1981.

[7] M. A. Virasoro, "Subsidiary conditions and ghosts in dualresonance models," Physical Review D, vol. 1, no. 10, pp. 29332936, 1970

[8] R. Hernández Heredero and E. G. Reyes, "Geometric integrability of the Camassa-Holm equation II," International Mathematics Research Notices, vol. 2012, no. 13, pp. 3089-3125, 2012.

[9] P. Górka and E. G. Reyes, "The modified Hunter-Saxton equation," Journal of Geometry and Physics, vol. 62, no. 8, pp. 17931809, 2012.

[10] M. Adler, "On a trace functional for formal pseudo-differential operators and the symplectic structure of the Korteweg-devries type equations," Inventiones Mathematicae, vol. 50, no. 3, pp. 219-248, 1978.

[11] B. Khesin, V. Lyubashenko, and C. Roger, "Extensions and contractions of the Lie algebra of $q$-pseudodifferential symbols on the circle," Journal of Functional Analysis, vol. 143, no. 1, pp. 55-97, 1997.

[12] F. Fathi-Zadeh and M. Khalkhali, "The algebra of formal twisted pseudodifferential symbols and a noncommutative residue," Letters in Mathematical Physics, vol. 94, no. 1, pp. 41-61, 2010.

[13] A. L. Pirozerski and M. A. Semenov-Tian-Shansky, "Generalized q-deformed Gelfand-Dickey structures on the group of q-pseudo difference operators," in Proceedings of the L.D. Faddeev's Seminar on Mathematical Physics, vol. 201 of AMS Translations Series 2, pp. 211-238, American Mathematical Society, Providence, RI, USA, 2000.

[14] A. L. Pirozerski and M. A. Semenov-Tian-Shansky, "Q-pseudodifference universal Drinfeld-Sokolov reduction," in Proceedings of the St. Petersburg Mathematical Society, vol. 7 of Series 2: American Mathematical Society, 203, pp. 169-199, AMS, Providence, RI, USA, 2001.

[15] V. G. Drinfel'd, "Quantum groups," in Proceedings of the ICM, vol. 1, pp. 798-820, American Mathematical Society, Berkeley, Calif, USA, 1986.

[16] O. S. Kravchenko and B. A. Khesin, "A central extension of the algebra of pseudodifferential symbols," Functional Analysis and Its Applications, vol. 25, no. 2, pp. 152-154, 1991.

[17] B. Khesin and R. Went, The Geometry of Infinite-Dimensional Groups, Springer, Berlin, Germany, 2009.

[18] B. A. Khesin, "A hierarchy of centrally extended algebras and the logarithm of the derivative operator," International Mathematics Research Notices, no. 1, pp. 1-5, 1992.

[19] E. E. Demidov, "On the Kadomtsev-Petviashvili hierarchy with a noncommutative timespace," Functional Analysis and Its Applications, vol. 29, no. 2, pp. 131-133, 1995. 
[20] E. E. Demidov, "Noncommutative deformation of the Kadomtsev-Petviashvili hierarchy," in Algebra. 5, Vseross. Inst. Nauchn. i Tekhn. Inform. (VINITI), Moscow, Russia, 1995, (Russian), Journal of Mathematical Sciences, vol. 88, no. 4, pp. 520-536, 1998, (English).

[21] K. Tenenblat, Transformations of Manifolds and Applications to Differential Equations, vol. 93 of Pitman Monographs and Surveys in Pure and Applied Mathematics, Longman, Harlow, UK, 1998.

[22] A. N. Parshin, "On a ring of formal pseudo-differential operators," Proceedings of the Steklov Institute of Mathematics, vol. 1, no. 224, pp. 266-280, 1999.

[23] A. S. Dzhumadil'daev, "Derivations and central extensions of the Lie algebra of formal pseudodifferential operators," Algebra i Analiz, vol. 6, no. 1, pp. 140-158, 1994.

[24] J. Beltran and M. Farinati, "The Hochschild and Lie (co)homology of the algebra of pseudodifferential operators in one and several variables," Preprint, http://www.complejidadsocial.cl/wpcontent/uploads/2015/05/HHPDiff-mod.pdf.

[25] M. Bordemann, "Nondegenerate invariant bilinear forms on nonassociative algebras," Acta Mathematica Universitatis Comenianae, vol. 66, no. 2, pp. 151-201, 1997.

[26] B. A. Khesin and I. Zakharevich, "The Lie-Poisson group of pseudodifferential symbols and fractional KP-KdV hierarchies," Comptes Rendus de l'Académie des Sciences. Serie I, vol. 316, no. 6, pp. 621-626, 1993.

[27] B. Khesin and I. Zakharevich, "Poisson-Lie group of pseudodifferential symbols," Communications in Mathematical Physics, vol. 171, no. 3, pp. 475-530, 1995.

[28] A. G. Reyman and M. A. Semenov-Tian-Shansky, "Current algebras and nonlinear partial differential equations," Soviet Mathematics-Doklady, vol. 251, no. 6, pp. 1310-1314, 1980.

[29] M. A. Semenov-Tyan-Shanskii, "What is a classical r-matrix?" Functional Analysis and Its Applications, vol. 17, no. 4, pp. 259272, 1983.

[30] Y. I. Manin, "Algebraic aspects of nonlinear differential equations," Journal of Soviet Mathematics, vol. 11, pp. 1-122, 1979 (Russian).

[31] M. Mulase, "Solvability of the super KP equation and a generalization of the Birkhoff decomposition," Inventiones Mathematicae, vol. 92, no. 1, pp. 1-46, 1988.

[32] D. B. Fuks, Cohomology of Infinite-Dimensional Lie Algebras, Contemporary Soviet Mathematics, Consultants Bureau, New York, NY, USA, 1986.

[33] A. S. Dzhumadil'daev, "Central extensions and invariant forms of Cartan type Lie algebras of positive characteristic," Functional Analysis and Its Applications, vol. 18, no. 4, pp. 331-332, 1984.

[34] A. S. Dzhumadil'daev, "Central extensions of infinite-dimensional Lie algebras," Functional Analysis and Its Applications, vol. 26, no. 4, pp. 247-253, 1992.

[35] A. I. Kostrikin and I. R. Šafarevič, "Graded Lie algebras of finite characteristic," Mathematics of the USSR-Izvestiya, vol. 3, no. 2, pp. 237-304, 1969.

[36] W. L. Li, "2-cocyles on the algebra of differential operators," Journal of Algebra, vol. 122, no. 1, pp. 64-80, 1989.

[37] M. Wodzicki, "Noncommutative residue chapter I. Fundamentals," in K-Theory, Arithmetic and Geometry: Seminar, Moscow University, 1984-1986, vol. 1289 of Lecture Notes in Mathematics, pp. 320-399, Springer, Berlin, Germany, 1987.

[38] A. Eslami Rad and E. G. Reyes, "The Kadomtsev-Petviashvili hierarchy and the Mulase factorization of formal Lie groups," Journal of Geometric Mechanics, vol. 5, no. 3, pp. 345-364, 2013. 


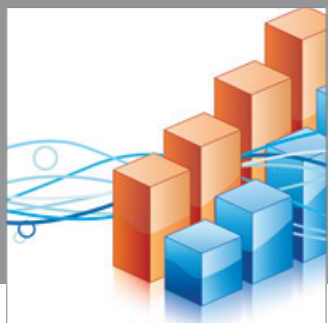

Advances in

Operations Research

mansans

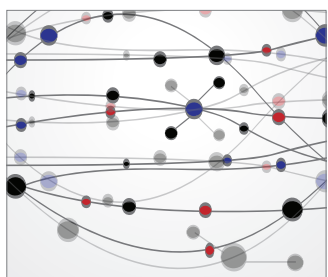

The Scientific World Journal
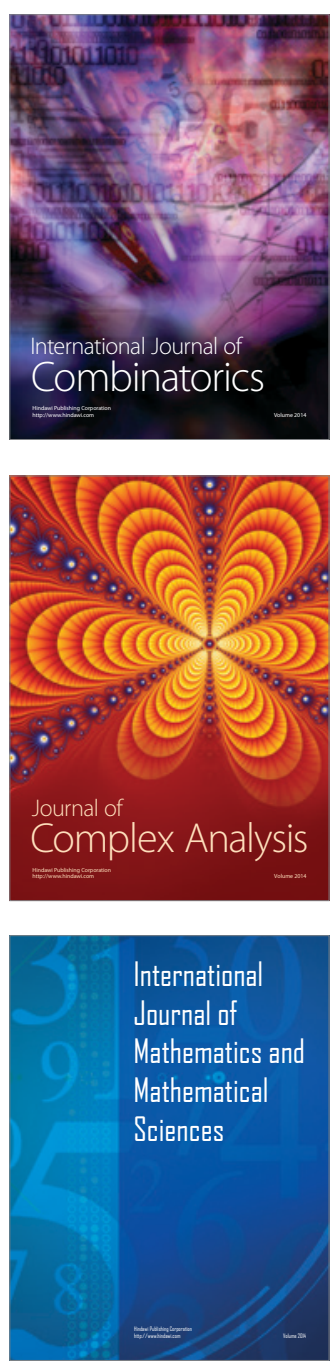
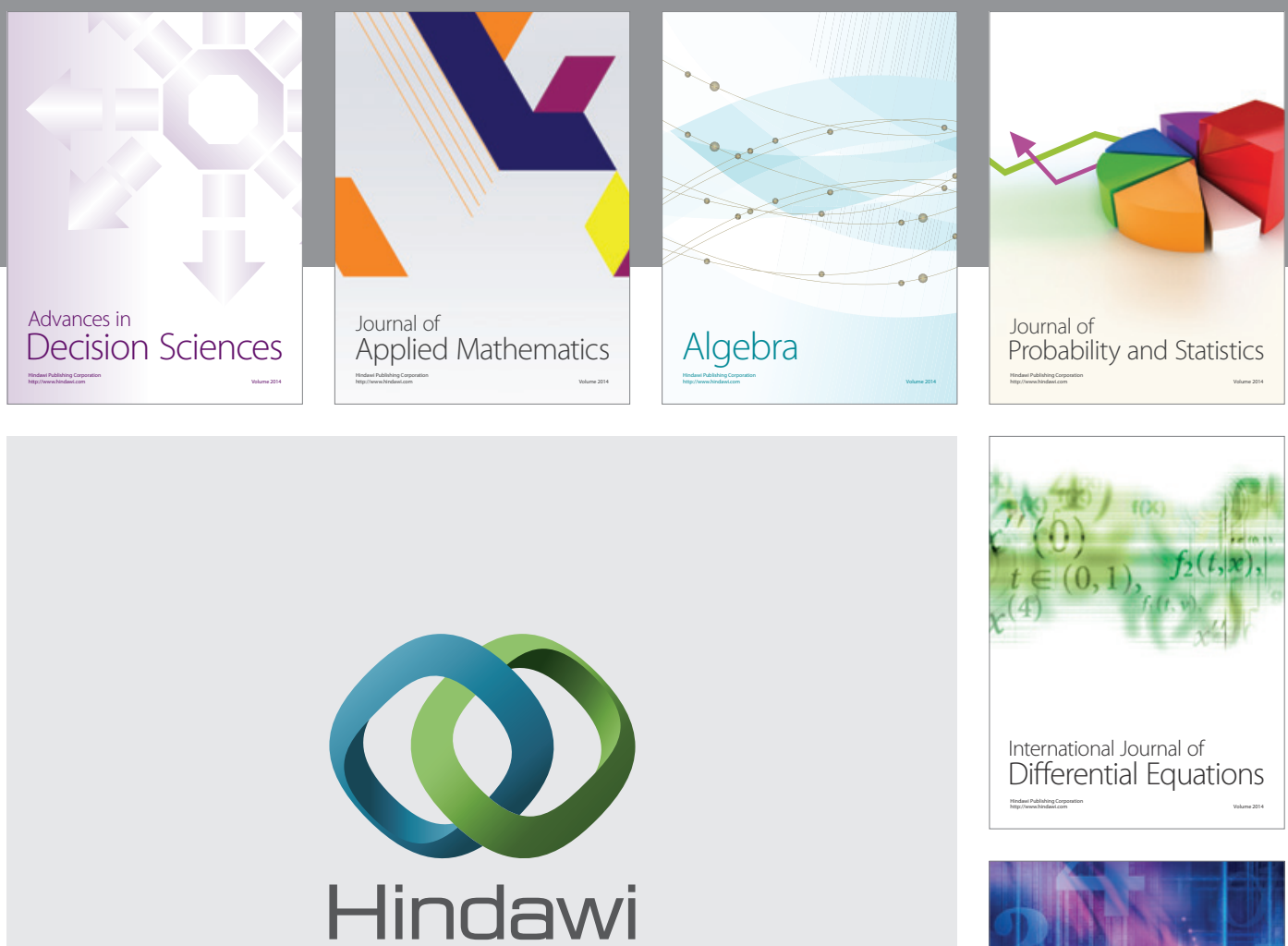

Submit your manuscripts at http://www.hindawi.com
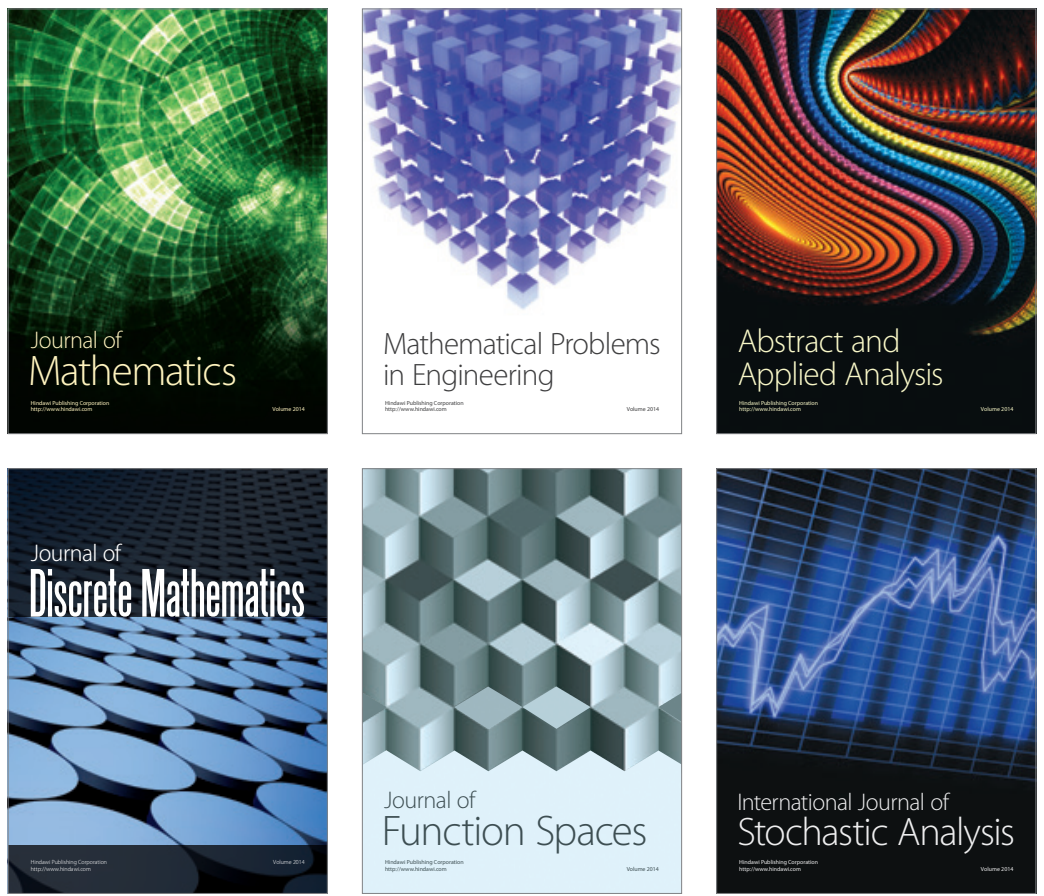

Journal of

Function Spaces

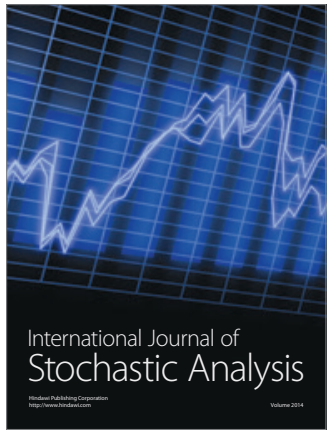

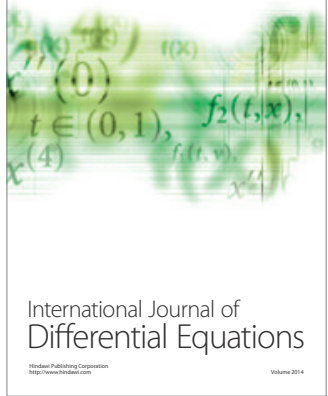
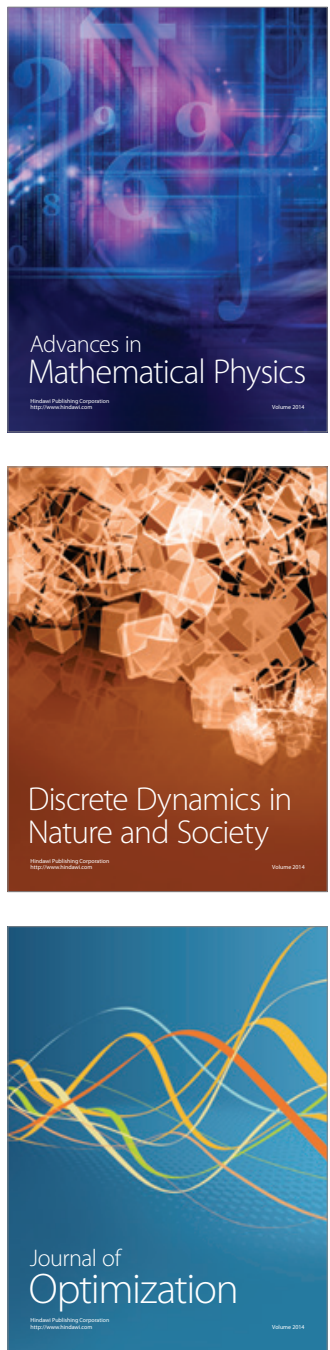